\title{
On DS-diagrams for 3-manifolds of Heegaard Genus 2
}

\author{
Mariko ENDOH
}

Sophia University

(Communicated by K. Shinoda)

\begin{abstract}
The block number $B l(M)$ introduced in our previous paper is a new topological invariant of a closed orientable 3-manifold $M$ which estimates a combinatorial complexity of $M$ just like the Heegaard genus $H G(M)$. In our previous paper, we have shown an inequality $H G(M) \leq B l(M)$ for any $M \neq S^{2} \times S^{1}$. In this paper, we will show that $B l(M)=H G(M)$ for any $M$ with $H G(M)=2$ and moreover that $B l(M) \leq 4$ for any $M$ with $H G(M)=3$.
\end{abstract}

\section{Introduction}

As a new topological invariant, we have introduced the block number for an orientable closed 3-manifold $M$, denoted by $B l(M)$, in [2]. The block number is defined through a DSdiagram with E-cycle (see [5]). In [2], we have shown that the block number $B l(M)$ dominates the Heegaard genus $H G(M)$ for any $M \neq S^{2} \times S^{1}$. Besides this, we have exhibited some other properties of the block number, for examples, $B l\left(S^{2} \times S^{1}\right)=0, B l\left(S^{3}\right)=1, B l(L(p, q))=$ $H G(L(p, q))=1, B l(M)=H G(M)=2$ for a Seifert fibered space $M$ having the 2 -sphere $S^{2}$ as its base manifold and three exceptional fibers. An interesting problem that remains to be considered is whether $H G(M)=B l(M)$ for any $M \neq S^{2} \times S^{1}, S^{3}$.

In this paper, we focus on the relation between the block number and the Heegaard genus for an oriented closed 3-manifolds. We will give a sufficient condition for $B l(M)=H G(M)$ in $\S 3$. However in the case of $H G(M)=g \geq 3$, it seems to be difficult to see if any manifold $M$ have a Heegaard diagram of genus $g$ which satisfies our sufficient condition. In $\$ 4$, by a technique of DS-diagrams which does not need the sufficient condition in $\S 3$, we make sure that a DS-diagram induced from any Heegaard-diagram with genus 2 can be deformed into the one with E-cycle whose block number is 2, that is, we will show the following main theorem of this paper.

THEOREM 1. $B l(M)=H G(M)=2$ for any orientable closed 3-manifold $M$ with $H G(M)=2$.

Applying the same method as our proof of Theorem 1, we obtain $B l(M) \leq 4$ for any $M$ with $H G(M)=3$ in $\S 5$. However it seems to be difficult to show whether $B l(M)=H G(M)$

Received October 8, 2004; revised February 24, 2005 


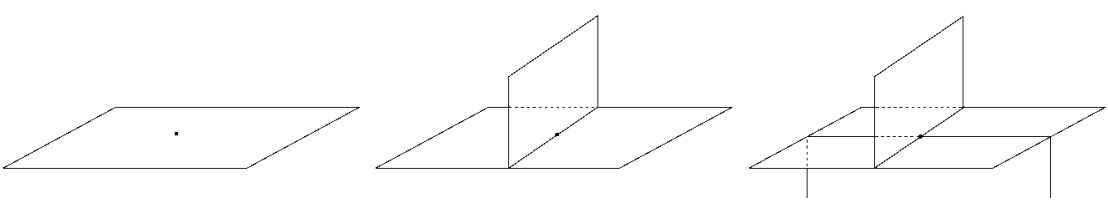

FIGURE 1 .

for any $M$ with $H G(M) \geq 3$. We will investigate what is the difficulty for proving $B l(M)=$ $H G(M)=3$.

The next section, $\S 2$, is devoted to review some definitions and some basic properties of the block number which we need in this paper.

\section{Preliminaries and definitions}

The notion of fake surfaces, DS-diagrams, and DS-diagrams with E-cycle were introduced by H. Ikeda ([3]-[5]). In this section, we shall review these concepts, and define the block number for an orientable closed 3-manifold.

2.1. Fake surfaces, simple spines and DS-diagrams. A finite 2-dimensional polyhedron $P$ is called a closed fake surface, if any point has a neighborhood homeomorphic to an open subset of

$$
\left(\mathbf{R}^{2} \times\{0\}\right) \cup\left(\mathbf{R} \times\{0\} \times \mathbf{R}_{+}\right) \cup\left(\{0\} \times \mathbf{R} \times \mathbf{R}_{-}\right) \subset \mathbf{R}^{3},
$$

that is, the shape of a neighborhood of a point $x \in P$ is one of the three types shown in Figure 1.

A closed fake surface $P$ is naturally stratified as $V(P) \subset S(P) \subset P$, where $V(P)$ is a finite set of points, called vertices, which play the role of 0 in the above subset of $\mathbf{R}^{3}$, and $S(P)$ is the singular set which consists of the points playing the role of $(t, 0,0)$ or $(0, t, 0)$. A connected component of $P-S(P)$ is called a face of $P$, and a connected component of $S(P)-V(P)$ is called an edge of $P$. In this paper, we assume that a face of a closed fake surface is an open 2-disk, and an edge is an open arc.

If a closed fake surface $P$ is embedded in a closed 3-manifold $M$, so that $M-P$ is homeomorphic to an open 3-ball $\mathbf{B}^{3}$, then we call $P$ a simple spine of $M$. For a simple spine $P$ of a closed 3-manifold $M$, cutting out along $P$, we obtain a 3 -ball $\mathbf{B}^{3}$ with a gluing map on its boundary $S^{2}=\partial \mathbf{B}^{3}$. We can formulate this situation as the following definition of a DS-diagram.

Definition 2.1.1. A triple $\Delta=(G, f, P)$ is called a $D S$-diagram if

(1) $G$ is a 3-regular graph embedded in the 2-sphere $S^{2}$,

(2) $P$ is a closed fake surface,

(3) $f$ is a local homeomorphism from $S^{2}$ onto $P$ such that $f^{-1}(S(P))=G$ and $f^{-1}(V(P))=V_{G}$, where $V_{G}$ is the set of the vertices of the graph $G$, and 
(4) $\# f^{-1}(x)=4$ for any $x \in V(P), \# f^{-1}(x)=3$ for any $x \in S(P)-V(P)$, and $\# f^{-1}(x)=2$ for any $x \in P-S(P)$.

Let $\mathbf{B}^{3}$ be a 3-ball with the boundary $\partial \mathbf{B}^{3}=S^{2}$. For a DS-diagram $\Delta=(G, f, P)$, gluing $\partial \mathbf{B}^{3}$ by the map $f$, we get a closed 3-manifold $M(\Delta)=\mathbf{B}^{3} / f$ with a simple spine $P=S^{2} / f$ of $M(\Delta)$. An element of $V_{G}$ is called a vertex of $\Delta$, a connected component of $S^{2}-G$ is called a face of $\Delta$ and a connected component of $G-V_{G}$ is called an edge of $\Delta$. For a face $\sigma$ of $P, f^{-1}(\sigma)$ consist of exactly two faces $\sigma^{+}$and $\sigma^{-}$of $\Delta$ such that $f\left(\sigma^{+}\right)=f\left(\sigma^{-}\right)=\sigma$. We say that $\sigma^{ \pm}$is a spouse of $\sigma^{\mp}$, respectively.

We always assume that the closed 3-manifold $M=M(\Delta)$ represented by a DS-diagram $\Delta$ is orientable.

2.2. DS-diagrams with E-cycle and E-data. A cycle $c=\left\{E_{1}, E_{2}, \cdots, E_{v}\right\}$ of a graph $G$ is a sequence of edges $E_{j}\left(E_{i} \neq E_{j}\right.$ if $\left.i \neq j\right)$ such that the closure of $\bigcup_{j} E_{j}$ is a simple closed curve.

Definition 2.2.1. Let $\Delta=(G, f, P)$ be a DS-diagram, $e$ be a cycle of $G$ (the underlying space of the closure of the cycle $e$ is denoted by the same letter $e$ ), and $H^{+}$and $H^{-}$ be the connected components of $S^{2}-e$. The cycle $e$ of the graph $G$ is said to be an $E$-cycle, if it satisfies that

(1) $\#\left(e \cap f^{-1}(x)\right)=2$ for any $x \in V(P)$,

(2) $\#\left(e \cap f^{-1}(x)\right)=1$ for any $x \in S(P)-V(P)$, and

(3) the restriction of $f$ onto $H^{+}$(or $H^{-}$) is a bijection.

There exists a DS-diagram which admits some different E-cycles. When we consider a DS-diagram with E-cycle, we always assume that an E-cycle $e$ is specified, and has a fixed orientation. Moreover we assume that $S^{2}$ on which the graph $G$ is drawn is oriented, and the component $H^{+}$of $S^{2}-e$ of which the restricted orientation is compatible with $e$, that is $\partial H^{+}=e$ (in the oriented sense), is called the positive region. We represent a DS-diagram with E-cycle by a four-tuple $\Delta=(G, f, P ; e)$. It is known that any closed 3-manifold can be represented by a DS-diagram with E-cycle ([5], [8]), and moreover it is known that, in the orientable case, a DS-diagram with E-cycle defines a closed manifold (see [8] and [9]).

Let $\Delta=(G, f, P ; e)$ be a DS-diagram with E-cycle, which is assumed to be given on the unit sphere in $\mathbf{R}^{3}$. For each vertex $v \in V(P)$ of the spine $P=\partial \mathbf{B}^{3} / f$, there are exactly two vertices $v^{+}$and $v^{-}$of the graph $G$ such that $f\left(v^{+}\right)=f\left(v^{-}\right)=v$, and $v^{+}$and $v^{-}$are both on the E-cycle $e$. These two vertices are characterized by the condition

(*) $\quad U \cap(G-e) \subset H^{+}$(or $H^{-}$) for sufficiently small neighborhood $U$ (in $S^{2}$ ) of $v^{+}$ (respectively $v^{-}$).

Each vertex $v \in V(P)$ is classified into the two cases $(\ell)$ or $(r)$ shown in Figure 2 (cf. [8] and [9]). We define the code $\phi(v)$ so that $\phi(v)=\ell$ (or $r$ ) if $v$ is the vertex of type $(\ell)$ (respectively (r)). 
$(\ell)$

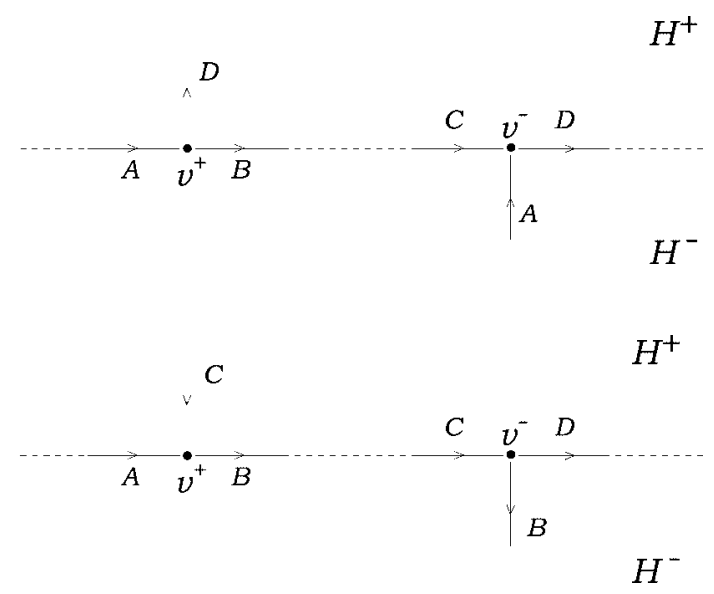

FIGURE 2.

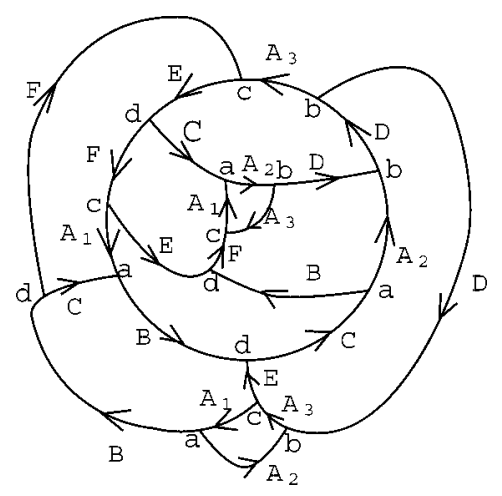

FIGURE 3.

Suppose that $V(P)$ consists of $n$ points $v_{1}, v_{2}, \cdots, v_{n}$. An E-data

$$
\mathcal{E}(\Delta)=(\phi, \mathcal{A}(\Delta))
$$

of $\Delta=(G, f, P ; e)$ is a pair of the code $\phi\left(v_{k}\right)$ for each $v_{k} \in V(P)$ and the arrangement $\mathcal{A}(\Delta)$ of $2 n$ points $v_{k}^{ \pm}$on the oriented circle $S^{1} \equiv e$.

It is known that an E-data completely determines a DS-diagram with E-cycle, and there are several methods for representing an E-data (see [8], [9], [10], and see also [1]). In this paper, we use a representation of an E-data by a coded sequence which is introduced in [2].

EXAmple 1. Let $\Delta=(G, f, P ; e)$ be the DS-diagram drawn in Figure 3, which has four vertices $V(P)=\{a, b, c, d\}$. The codes of these vertices are $\phi(a)=\phi(c)=\phi(d)=\ell$ 
and $\phi(b)=r$, and the arrangement $\mathcal{A}(\Delta)$ is given by a sequence

$$
\mathcal{A}(\Delta)=\left\{a^{+} b^{+} b^{-} c^{-} d^{+} c^{+} a^{-} d^{-}\right\},
$$

which indicates the cyclical order of the points in $V(G) \cap e$. Of course we may employ a cyclically permuted sequence for representing the arrangement. The E-data $\mathcal{E}(\Delta)$ can be represented by the following coded sequence:

$$
\mathcal{E}(\Delta)=\left\{a^{+\ell} b^{+r} b^{-r} c^{-\ell} d^{+\ell} c^{+\ell} a^{-\ell} d^{-\ell}\right\} .
$$

We call a consecutive (in the sense of cyclical order) sequence of symbols in $\mathcal{A}(\Delta)$ a subword of an arrangement $\mathcal{A}(\Delta)$. If any symbol in a subword $W$ has the signature $+($ or - ), then we call $W$ a positive subword (respectively a negative subword).

Definition 2.2.2. A maximal positive (or negative) subword of an arrangement $\mathcal{A}(\Delta)$ is called a positive block (respectively a negative block) of $\mathcal{A}(\Delta)$.

For example, the arrangement $\mathcal{A}(\Delta)$ in Example 1 has two positive blocks $W_{1}^{+}=a^{+} b^{+}$, $W_{2}^{+}=d^{+} c^{+}$and two negative blocks $W_{3}^{-}=b^{-} c^{-}, W_{4}^{-}=a^{-} d^{-}$, and we can represent $\mathcal{A}(\Delta)$ as $\mathcal{A}(\Delta)=W_{1}^{+} W_{3}^{-} W_{2}^{+} W_{4}^{-}$. Also for a coded sequence we can define the notion of a "subword", a "positive subword" and a "negative subword".

The block number of a DS-diagram with E-cycle of an orientable closed 3-manifold are defined as follow.

Definition 2.2.3. The block number $b l(\Delta)$ of a DS-diagram $\Delta$ with E-cycle is defined to be the number of positive blocks included in the arrangement $\mathcal{A}(\Delta)$.

2.3. Moves for E-data and block number of a 3-manifold. The moves for DSdiagrams with E-cycle, generators of the deformations of DS-diagrams with E-cycle which preserve the represented manifold, were introduces in [9]. Here we will review those moves in terms of the coded sequences for E-data.

DEFINITION 2.3.1 (The first regular move $R_{1}$ ). Let $\Delta$ be a DS-diagram with E-cycle whose E-data $\mathcal{E}(\Delta)$ includes three subwords $W_{1}=a^{-\ell} b^{+\ell}, W_{2}=a^{+\ell} x^{+\ell}$ and $W_{3}=$ $x^{-\ell} b^{-\ell}$. Then $R_{1}$ is defined to be a deformation changing $\Delta$ into $\Delta^{\prime}$ with the E-data $\mathcal{E}\left(\Delta^{\prime}\right)$ in which the subwords $W_{k}(k=1,2,3)$ are replaced by $W_{1}^{\prime}=b^{+\ell} a^{-\ell}, W_{2}^{\prime}=a^{+\ell}$ and $W_{3}^{\prime}=b^{-\ell}$ respectively. By this move, $a^{-\ell}$ and $b^{+\ell}$ mutually exchange their places in the coded sequence, and $x^{ \pm \ell}$ are eliminated. Hence, for the spines $P$ represented by $\Delta$ and $P^{\prime}$ represented by $\Delta^{\prime}$, we have $\# V(P)=\# V\left(P^{\prime}\right)+1$.

DEFINITION 2.3.2 (The second regular move $R_{2}$ ). Let $\Delta$ be a DS-diagram with Ecycle whose E-data $\mathcal{E}(\Delta)$ includes two subwords $W_{1}=x^{-\ell} y^{-r}$ (or $y^{-r} x^{-\ell}$ ) and $W_{2}=$ $x^{+\ell} y^{+r}$ (or $y^{+r} x^{+\ell}$ ). Then $R_{2}$ is defined to be a deformation eliminating these two subwords $W_{1}$ and $W_{2}$. Hence, for two spines $P$ represented by $\Delta$ and $P^{\prime}$ represented by $\Delta^{\prime}=R_{2}(\Delta)$

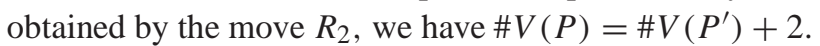




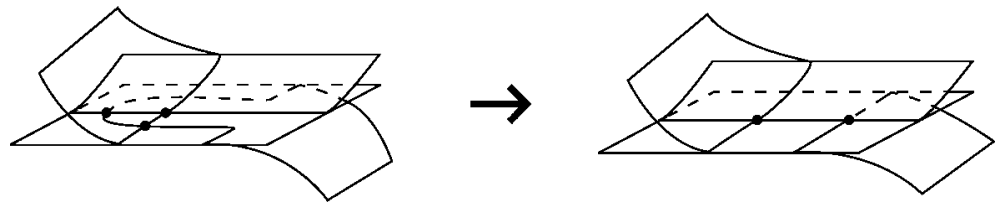

Figure 4. The first regular move $R_{1}$.

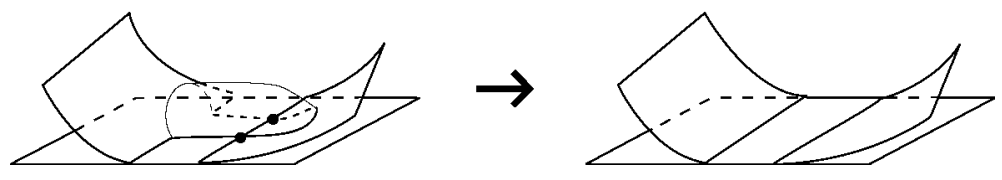

FIGURE 5. The second regular move $R_{2}$.

The inverses of the above moves $R_{j}$ are denoted by $R_{j}^{-1}$. If there is a sequence $\Delta=$ $\Delta_{0}, \Delta_{1}, \Delta_{2}, \cdots \Delta_{n}=\Delta^{\prime}$ of DS-diagrams with E-cycle such that $\Delta_{k}$ is obtained from $\Delta_{k-1}$ by one of the moves $R_{1}^{ \pm 1}$ and $R_{2}^{ \pm 1}$, then $\Delta^{\prime}$ is said to be regularly equivalent to $\Delta$, denoted by $\Delta^{\prime} \cong \Delta$.

Another move, called the surgery move, is defined as follows.

DEFINITION 2.3.3 (The surgery move $S$ ). If $\Delta$ is a DS-diagram with E-cycle whose E-data $\mathcal{E}(\Delta)$ includes a subword $W=x^{-\ell} y^{+\ell} z^{+\ell} z^{-\ell} w^{-r} y^{-\ell} w^{+r} x^{+\ell}$, then the surgery move $S$ gives a DS-diagram $\Delta^{\prime}=S(\Delta)$ with the E-data $\mathcal{E}\left(\Delta^{\prime}\right)$ obtained from $\mathcal{E}(\Delta)$ by eliminating the subword $W$.

If there is a sequence $\Delta=\Delta_{0}, \Delta_{1}, \Delta_{2}, \cdots \Delta_{n}=\Delta^{\prime}$ of DS-diagrams with E-cycle such that $\Delta_{k}$ is obtained from $\Delta_{k-1}$ by one of the moves $R_{1}^{ \pm 1}, R_{2}^{ \pm 1}$ and $S^{ \pm 1}$, then $\Delta^{\prime}$ is said to be equivalent to $\Delta$, denoted by $\Delta^{\prime} \sim \Delta$.

The following theorem was shown in [9].

THEOREM 2. Let $\Delta$ and $\Delta^{\prime}$ be DS-diagrams with E-cycle. Then there is orientation preserving homeomorphism from $M(\Delta)$ onto $M\left(\Delta^{\prime}\right)$ if and only if $\Delta \sim \Delta^{\prime}$.

DEFINITION 2.3.4. Let $M$ be a orientable closed 3-manifold. Let $\Delta$ be a DS-diagram with E-cycle representing $M(\Delta)=M$. The block number $B l(M)$ of an orientable closed 3-manifold $M$ is defined by

$$
B l(M)=\min \left\{b l\left(\Delta^{\prime}\right) \mid \Delta^{\prime} \sim \Delta\right\} .
$$

Obviously $B l(M)$ is a topological invariant for closed orientable 3-manifolds. The next proposition was shown in [2].

Proposition 1. $H G(M) \leq B l(M)$ for any closed orientable 3-manifold $M$ except for $S^{2} \times S^{1}$. 


\section{A sufficient condition for $B l(M)=H G(M)$}

In this section we will give a sufficient condition for a Heegaard diagram of a closed 3-manifold $M$ with genus $g$ which guarantees the existence of a DS-diagram $\Delta$ with E-cycle such that $M(\Delta) \cong M$ and $b l(\Delta)=g$.

Let $M$ be a closed 3-manifold having a Heegaard diagram $\left(M_{1}, M_{2} ; \vec{D}_{1}, \vec{D}_{2}\right)$ with genus $g$, where $M_{1}$ and $M_{2}$ are handlebodies such that $\partial M_{1}=\partial M_{2}$ and $M=M_{1} \cup M_{2}$ with a gluing map $h: \partial M_{2} \rightarrow \partial M_{1}$, and $\vec{D}_{1}=\left\{\alpha_{1}, \alpha_{2}, \cdots \alpha_{g}\right\}$ and $\vec{D}_{2}=\left\{\beta_{1}, \beta_{2}, \cdots \beta_{g}\right\}$ are complete meridian disk systems of $M_{1}$ and $M_{2}$ respectively. Deforming the meridian disks $\vec{D}_{1} \cup \vec{D}_{2}$, if necessary, we may assume that $\partial \vec{D}_{1} \cup \partial \vec{D}_{2}$ is connected and each connected components of $\partial M_{i}-\left(\partial \vec{D}_{1} \cup \partial \vec{D}_{2}\right)(i=1,2)$ is an open 2-cell. Then the union $\tilde{P}=F \cup \vec{D}_{1} \cup \vec{D}_{2}$ of the Heegaard surface $F \equiv \partial M_{i}$ and the meridian disks in the systems $\vec{D}_{i}(i=1,2)$ forms a closed fake surface embedded in $M$, whose singularity $S(\tilde{P})$ consists of meridian curves $\partial \vec{D}_{1} \equiv \bigcup \partial \alpha_{i}$ and $\partial \vec{D}_{2} \equiv \bigcup \partial \beta_{i}$, and whose vertices $V(\tilde{P})$ consists of $\vec{D}_{1} \cap \vec{D}_{2}$. This fake surface $\tilde{P}$ is not a simple spine because its complement is a union of two open 3-balls $M_{1}-\vec{D}_{1}$ and $M_{2}-\vec{D}_{2}$. A face of the fake surface $\tilde{P}$ is either one of meridian disks $\alpha_{i}$ and $\beta_{i}$ or a connected component of $F-\left(\partial \vec{D}_{1} \cup \partial \vec{D}_{2}\right)$. Removing a face $\lambda$ of $\tilde{P}$ which is on $F$ and whose closure is a compact 2-disk, we can obtain a simple spine $P=\tilde{P}-\lambda$ which we call a derived spine. However the DS-diagram of a derived spine is not necessarily one with E-cycle. In what follows we will show that we obtain a DS-diagram with E-cycle if the face to be removed has some good property.

Definition 3.0.1 (A good $2 g$-gon). A face $\lambda_{0}$ of the fake surface $\tilde{P}$ is said to be a good $2 g$-gon if

(1) the closure of $\lambda_{0}$ is a compact 2-disk,

(2) $V(\tilde{P}) \cap \partial \lambda_{0}$ consists of exactly $2 g$ points, and

(3) any two edges on $\partial \lambda_{0}$ are on mutually different meridian, that is, $\partial \lambda_{0} \cap \partial \alpha_{i} \neq \emptyset$ and $\partial \lambda_{0} \cap \partial \beta_{i} \neq \emptyset$ for any $i=1,2, \cdots, g$.

THEOREM 3. If the closed fake surface $\tilde{P}=F \cup \vec{D}_{1} \cup \vec{D}_{2}$ given by a Heegaard diagram of genus $g$ admits a good $2 g$-gon $\lambda_{0}$, then the derived simple spine $P \equiv \tilde{P}-\lambda_{0}$ has an E-cycle, and its DS-diagram $\Delta$ satisfies bl $(\Delta)=g$.

Proof. For simplicity, we will give the proof only for the case $H G(M)=2$. We can prove the lemma for general cases by a quite similar method.

Let $\left(M_{1}, M_{2} ; \vec{D}_{1}, \vec{D}_{2}\right)$ be a genus-2 Heegaard diagram of $M$, where $\vec{D}_{1}=\left\{\alpha_{1}, \alpha_{2}\right\}$ and $\vec{D}_{2}=\left\{\beta_{1}, \beta_{2}\right\}$ are complete meridian disk systems for the handlebodies $M_{1}$ and $M_{2}$ respectively. By $h$ we denote the gluing map $h: \partial M_{2} \rightarrow \partial M_{1}$.

Assume $\lambda_{0}$ to be a good 4-gon on the closed fake surface $\tilde{P}=F \cup \vec{D}_{1} \cup \vec{D}_{2}$, and consider the derived spine $P=\tilde{P}-\lambda_{0}$. The DS-diagram $\Delta=(G, f, P)$ of the derived 

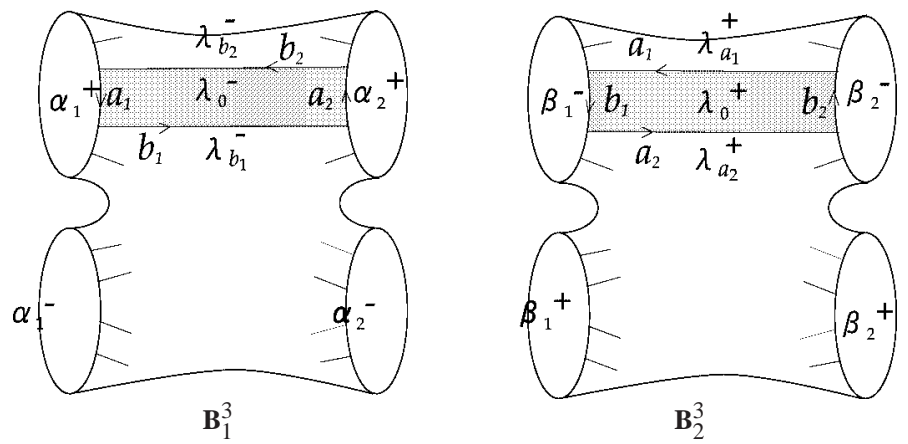

Figure 6. $c l(M-N(\tilde{P}))$.

spine $P$ can be obtained in the following manner. Let $\mathbf{B}_{i}^{3}(i=1,2)$ be the 3-ball obtained by cutting the handlebody $M_{i}$ along the meridian disks in the system $\vec{D}_{i}$. The gluing map $h$ and the cutting off operation naturally define the identification map $\tilde{h}: \partial \mathbf{B}_{1}^{3} \cup \partial \mathbf{B}_{2}^{3} \rightarrow \tilde{P}$. For a face $\lambda$ of $\tilde{P}$ which is on the Heegaard surface $F$, one of the components of $\tilde{h}^{-1}(\lambda)$ is on $\partial \mathbf{B}_{1}^{3}$, denoted by $\lambda^{-}$, and the other is on $\partial \mathbf{B}_{2}^{3}$, denoted by $\lambda^{+}$. The two components of $\tilde{h}^{-1}\left(\alpha_{i}\right)$ which we denote by $\alpha_{i}^{-}$and $\alpha_{i}^{+}$, are both on $\partial \mathbf{B}_{1}^{3}$, and the two components of $\tilde{h}^{-1}\left(\beta_{i}\right)$, which we denote by $\beta_{i}^{-}$and $\beta_{i}^{+}$, are both on $\partial \mathbf{B}_{2}^{3}$. Since $\lambda_{0}$ is a good 4-gon, we may assume that the inverse images $\lambda_{0}^{-}$and $\lambda_{0}^{+}$are situated on $\partial \mathbf{B}_{i}^{3}$ just as in Figure 6 . That is, $\lambda_{0}^{-}$ is adjacent to four faces $\alpha_{1}^{+}, \lambda_{b_{1}}^{-}, \alpha_{2}^{+}$, and $\lambda_{b_{2}}^{-}$where $\partial \lambda_{0}^{-} \cap \partial \alpha_{1}^{+}=a_{1}, \partial \lambda_{0}^{-} \cap \partial \lambda_{b_{1}}^{-}=b_{1}$, $\partial \lambda_{0}^{-} \cap \partial \alpha_{2}^{+}=a_{2}$ and $\partial \lambda_{0}^{-} \cap \partial \lambda_{b_{2}}^{-}=b_{2}$. And $\lambda_{0}^{+}$is adjacent to four faces $\lambda_{a_{1}}^{+}, \beta_{1}^{-}, \lambda_{a_{2}}^{+}$and $\beta_{2}^{-}$ where $\partial \lambda_{0}^{+} \cap \partial \lambda_{a_{1}}^{+}=a_{1}, \partial \lambda_{0}^{+} \cap \partial \beta_{1}^{-}=b_{1}, \partial \lambda_{0}^{+} \cap \partial \lambda_{a_{2}}^{+}=a_{2}$ and $\partial \lambda_{0}^{+} \cap \partial \beta_{2}^{-}=b_{2}$. Gluing $\lambda_{0}^{-}$ and $\lambda_{0}^{+}$, we obtain a 3-ball $\mathbf{B}^{3}$ (see Figure 7 and 8 ), on whose boundary $\partial \mathbf{B}^{3}$ the identification map $f: \partial B^{3} \rightarrow P=\tilde{P}-\lambda_{0}$ is naturally defined. Together with the graph $G=f^{-1}(S(P))$ on $\partial \mathbf{B}^{3}$, the triple $\Delta=(G, f, P)$ becomes the DS-diagram for the derived spine $P$.

After gluing $\lambda_{0}^{-}$and $\lambda_{0}^{+}$, the edges $a_{1}, b_{1}, a_{2}$ and $b_{2}$ of $\tilde{P}$ are not edges of $P$ any more. Two faces $\alpha_{1}^{+}$of $\mathbf{B}_{1}^{3}$ and $\lambda_{a_{1}}^{+}$of $\mathbf{B}_{2}^{3}$ become one face of $\Delta$. We denote this face by $\alpha_{1}^{+}$. The faces of $\Delta$ which result from $\alpha_{i}^{ \pm}$and $\lambda_{a_{i}}^{ \pm}$(or $\beta_{i}^{ \pm}$and $\lambda_{b_{i}}^{ \pm}$) of $\mathbf{B}_{1}^{3} \cup \mathbf{B}_{2}^{3}$ are denoted by $\alpha_{i}^{ \pm}$(or respectively $\left.\beta_{i}^{ \pm}\right)(i=1,2)$. Consider the cycle $e=\left(\partial \alpha_{1}^{+}-a_{1}\right) \cup\left(\partial \beta_{1}^{-}-b_{1}\right) \cup\left(\partial \alpha_{2}^{+}-\right.$ $\left.a_{2}\right) \cup\left(\partial \beta_{2}^{-}-b_{2}\right)$ of the graph $G$. The component of $S^{2}-e$ which includes the faces $\alpha_{i}^{-}, \beta_{i}^{-}$ is denoted by $H^{-}$. The other component, which consists of the faces $\alpha_{i}^{+}$and $\beta_{i}^{+}$and $\lambda_{j}^{+}$, is denoted by $H^{+}$. The restriction of $f$ onto $H^{+}$(or $H^{-}$) is a bijection. Since the singularity $S(P)$ consists of $\partial \vec{D}_{1} \cup \partial \vec{D}_{2}-\partial \lambda$, each edge $X$ on $P$ belongs to $\left(\partial \alpha_{i}-a_{i}\right)$ or $\left(\partial \beta_{j}-b_{j}\right)$. Three edges $f^{-1}(X)$ of $G$ appear on $e, H^{-}$and $H^{+}$. Since the vertices $V(P)$ consists of $\partial \vec{D}_{1} \cap \partial \vec{D}_{2}-\lambda$, each vertex $v$ of $P$ belongs to $\left(\partial \alpha_{i}-a_{i}\right) \cap\left(\partial \beta_{j}-b_{j}\right)$. Four vertices $f^{-1}(v)$ 


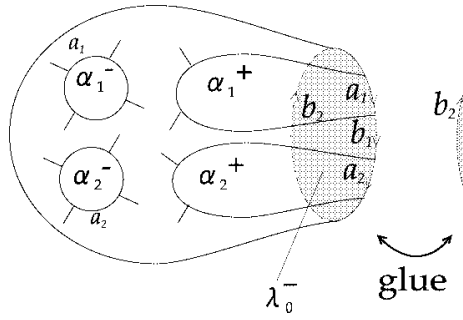

$\mathbf{B}_{1}^{3}$

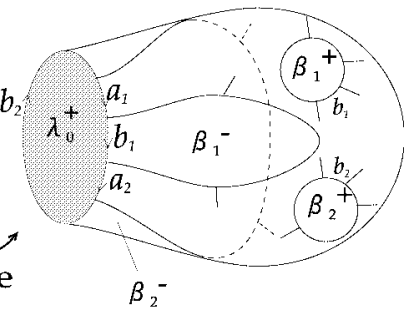

$\mathbf{B}_{2}^{3}$

FIGURE 7. gluing $\mathbf{B}_{1}^{3}$ and $\mathbf{B}_{2}^{3}$.

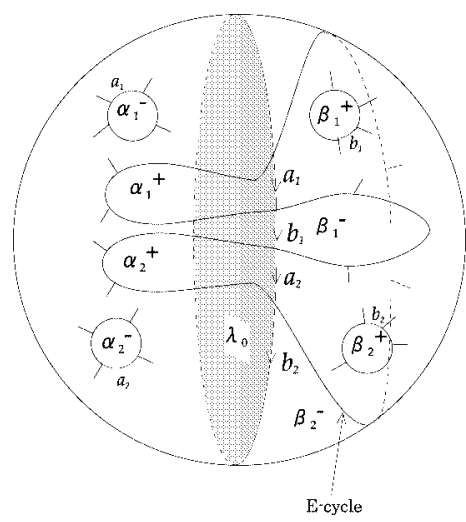

FIGURE 8. A DS-diagram $\Delta$ with E-cycle.

of $G$ appear on $\partial \alpha_{i}^{+}-a_{i} \subset e, \partial \beta_{j}^{-}-b_{j} \subset e, \partial \alpha_{i}^{-}-a_{i} \subset H^{-}$and $\partial \beta_{j}^{+}-b_{j} \subset H^{+}$. So the cycle $e$ turns out to be an E-cycle of $\Delta$.

We can see that $b l(\Delta)=2$ because, for any vertices $v^{+}$and $v^{-}$on $e, v^{+}$is included in $\partial \beta_{1}^{-}-b_{1}$ or $\partial \beta_{2}^{-}-b_{2}, v^{-}$is included in $\partial \alpha_{1}^{+}-a_{1}$ or $\partial \alpha_{2}^{+}-a_{2}$.

Maybe our main theorem can be proved by applying the above theorem, that is, we can deform the meridian disk systems of any Heegaard diagram with genus 2 so that the deformed diagram has a good 4-gon. However it seems to be hard to find a good $2 g$-gon for a general Heegaard diagram with genus $g \geq 3$. So, in order to see what is the obstruction for getting a DS-diagram with the block number $g$ by a Heegaard diagram with genus $g$, we will try to prove the main theorem by using the remodeling algorithm in [7], which gives an algorithm for obtaining a DS-diagram with E-cycle from a given DS-diagram without E-cycle. In $\S 5$, we will show that a similar method gives an estimate $B l(M) \leq 4$ for any $M$ with $H G(M)=3$, and moreover we will explain what is the difficulty for proving $B l(M)=H G(M)$ in the case of $H G(M)=3$. 


\section{Proof of the main theorem}

In this chapter, $M$ is assumed to be an orientable closed 3-manifold with genus 2 .

The proof of the main theorem is divided into the following three steps:

step 1 ; defining a derived spine $P_{0} \equiv \tilde{P}-\lambda_{0}$,

step 2 ; remodeling $\Delta_{0}$ of $P_{0}$ into another DS-diagram $\Delta_{1}$ having an E-cycle,

step 3; reducing the block number of $\Delta_{1}$ to 2 by applications of moves.

The remodeling algorithm in step 2 needs mark lines, each of which is a proper arc on a face of the spine $P_{0}$ (see $\S 4.1$ below and also [7]), and, for the successful operation in step 3, these mark lines must be carefully chosen. Also the face $\lambda_{0}$ of the closed fake surface $\tilde{P}$ is so chosen that we can take desirable mark lines on the derived spine $P_{0} \equiv \tilde{P}-\lambda_{0}$.

4.1. A DS-diagram of a derived spine $P$. Let $\left(M_{1}, M_{2} ; \vec{D}_{1}, \vec{D}_{2}\right)$ be a Heegaard diagram with genus 2 giving a closed 3-manifold $M$. Deforming the meridian disks $\vec{D}_{1} \cup \vec{D}_{2}$ if necessary, we may assume that $\partial \vec{D}_{1} \cup \partial \vec{D}_{2}$ is connected and each connected components of $\partial M_{i}-\left(\partial \vec{D}_{1} \cup \partial \vec{D}_{2}\right)(i=1,2)$ is an open 2-cell. The notations $M_{i}, \vec{D}_{i}, \mathbf{B}_{i}^{3}, F, \tilde{P}$ and $\tilde{h}$ follow ones in $\S 3$. Let $\lambda_{0}, \lambda_{1}$ and $\lambda_{1}^{\prime}$ be three faces of the fake surface $\tilde{P}$ such that

(1) three faces $\lambda_{0}, \lambda_{1}$ and $\lambda_{1}^{\prime}$ are included in $F \subset \tilde{P}$,

(2) a face $\lambda_{0}$ is a 2-gon face whose inverse image $\lambda_{0}^{-}$is adjacent to $\alpha_{2}^{+}$, and the other inverse image $\lambda_{0}^{+}$is adjacent to $\beta_{2}^{-}$,

(3) the inverse image $\lambda_{1}^{-}$is adjacent to both $\lambda_{0}^{-}$and $\alpha_{1}^{+}$,

(4) the inverse image $\lambda_{1}^{\prime+}$ is adjacent to $\beta_{1}^{-}$and $\beta_{2}^{-}$.

The inverse images $\lambda_{0}^{-}, \lambda_{1}^{-}$and $\lambda_{1}^{\prime-}$ on $\partial \mathbf{B}_{1}^{3}$ and $\lambda_{0}^{+}, \lambda_{1}^{+}$and $\lambda_{1}^{\prime+}$ on $\partial \mathbf{B}_{2}^{3}$ are situated just as in Figure 9. Let $x, y, A, B_{1}, B_{2}, C_{1}, C_{2}, A^{\prime}$ and $B^{\prime}$ be edges of $\tilde{P}$ where $y=\partial \lambda_{0} \cap \partial \alpha_{2}$, $x=\partial \lambda_{0} \cap \partial \beta_{2}, A \subset \tilde{h}\left(\partial \lambda_{1}^{-} \cap \partial \alpha_{1}^{+}\right), A^{\prime} \subset \tilde{h}\left(\partial \lambda_{1}^{\prime+} \cap \partial \beta_{1}^{-}\right), B^{\prime} \subset \tilde{h}\left(\partial \lambda_{1}^{\prime+} \cap \partial \beta_{2}^{-}\right), \partial \alpha_{2}=$ $\cdots B_{1} y B_{2} \cdots$ and $\partial \beta_{2}=\cdots C_{1} x C_{2} \cdots B^{\prime} \cdots$.

Let $p \in A, q \in B_{2}, p^{\prime} \in A^{\prime}$ and $q^{\prime} \in B^{\prime}$ be four points. We call a pair of simple arcs $\gamma_{1}^{-}$ on $\partial \mathbf{B}_{1}^{3}$ and $\gamma_{2}^{+}$on $\partial \mathbf{B}_{2}^{3}$ pre mark-lines, if they satisfy that

(1) $\gamma_{1}^{-}$is a directed arc on $\lambda_{1}^{-}$such that $\tilde{h}\left(\gamma_{1}^{-}\right)$goes from $p$ to $q$,

(2) $\gamma_{2}^{+}$is a directed arc on $\lambda_{1}^{\prime+}$ such that $\tilde{h}\left(\gamma_{2}^{+}\right)$goes from $p^{\prime}$ to $q^{\prime}$ and

(3) $\tilde{h}\left(\gamma_{1}^{-}\right) \cap \tilde{h}\left(\gamma_{2}^{+}\right)=\emptyset$.

These pre mark-lines become mark-lines in step 2.

Note that, for $\gamma_{1}^{-}\left(\right.$or $\left.\gamma_{2}^{+}\right)$, there is the spouse $\gamma_{1}^{+}$(or $\left.\gamma_{2}^{-}\right)$such that $\tilde{h}\left(\gamma_{1}^{-}\right)=\tilde{h}\left(\gamma_{1}^{+}\right)=\gamma_{1}$ (respectively $\left.\tilde{h}\left(\gamma_{2}^{+}\right)=\tilde{h}\left(\gamma_{2}^{-}\right)=\gamma_{2}\right)$, see Figure 9 .

LEMMA 4.1.1. There exists a Heegaard diagram of $M$ which gives a closed fake surface admitting a 2-gon $\lambda_{0}$ and pre mark-lines $\gamma_{1}$ and $\gamma_{2}$. 


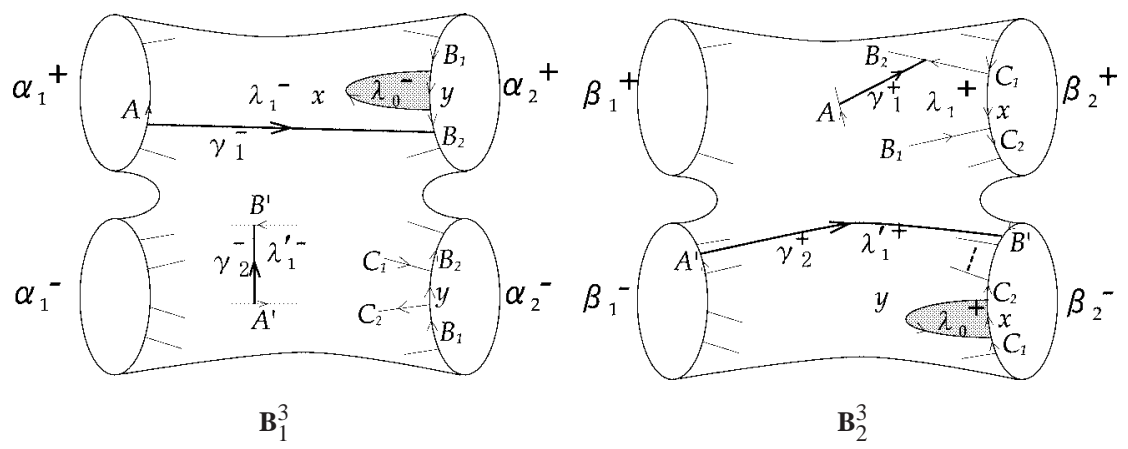

FIGURE 9. The pre mark-lines $\gamma_{1}^{-}$and $\gamma_{2}^{-}$on $\partial \mathbf{B}_{1}^{3}$, and $\gamma_{1}^{+}$and $\gamma_{2}^{+}$on $\partial \mathbf{B}_{2}^{3}$.

The proof of Lemma 4.1.1 will be shown in $\$ 4.5 .1$. We obtain a derived simple spine $P_{0} \equiv \tilde{P}-\lambda_{0}$ whose singularity $S\left(P_{0}\right)$ consists of $\partial \alpha_{1} \cup\left(\partial \alpha_{2}-y\right)$ and $\partial \beta_{1} \cup\left(\partial \beta_{2}-x\right)$, and whose vertices $V\left(P_{0}\right)$ consists of $\partial \vec{D}_{1} \cap \partial \vec{D}_{2}-\partial \lambda_{0}$. The diagram of Figure 10 is a DS-diagram of a derived spine $P_{0}=\tilde{P}-\lambda_{0}$. We denote this DS-diagram by $\Delta_{0}$. But the DS-diagram $\Delta_{0}$ have no E-cycle in general. So we will deform $\Delta_{0}$ into one with E-cycle by applying the algorithm of [7].

4.2. Remodeling a DS-diagram into one with E-cycle. Applying the remodeling algorithm to the DS-diagram $\Delta_{0}$ of the derived spine $P_{0}$ in the previous subsection, we construct a DS-diagram $\Delta_{1}$ with E-cycle. The algorithm is carried out along the mark-lines $\gamma_{1}$ and $\gamma_{2}$, and this operation produces some new vertices. These new vertices of the spine $P_{1}$ represented by $\Delta_{1}$ will be denoted by

$$
a_{i}(1 \leq i \leq 12), \quad b_{i}(1 \leq i \leq 9), \quad c_{i}(1 \leq i \leq p), \quad d_{i}(1 \leq i \leq p),
$$

where the number $p$ depends on the initial Heegaard diagram. The set $V\left(P_{1}\right)$ of vertices of $P_{1}$ consists of these new vertices and the original vertices $V\left(P_{0}\right)$. We can see (cf. §4.5) that the codes of the new vertices are

$$
\begin{aligned}
& \phi\left(a_{1}\right)=\ell, \quad \phi\left(a_{2}\right)=r, \quad \phi\left(a_{3}\right)=r, \quad \phi\left(a_{4}\right)=\ell, \quad \phi\left(a_{5}\right)=\ell, \quad \phi\left(a_{6}\right)=r, \\
& \phi\left(a_{7}\right)=r, \quad \phi\left(a_{8}\right)=\ell, \quad \phi\left(a_{9}\right)=\ell, \quad \phi\left(a_{10}\right)=r, \quad \phi\left(a_{11}\right)=\ell, \quad \phi\left(a_{12}\right)=r, \\
& \phi\left(b_{1}\right)=r, \quad \phi\left(b_{2}\right)=r, \quad \phi\left(b_{3}\right)=r, \quad \phi\left(b_{4}\right)=\ell, \quad \phi\left(b_{5}\right)=r, \quad \phi\left(b_{6}\right)=r, \\
& \phi\left(b_{7}\right)=\ell, \quad \phi\left(b_{8}\right)=\ell, \quad \phi\left(b_{9}\right)=r, \\
& \phi\left(c_{1}\right)=r, \quad \phi\left(c_{p}\right)=\ell, \\
& \phi\left(d_{1}\right)=\ell, \quad \phi\left(d_{p}\right)=r .
\end{aligned}
$$




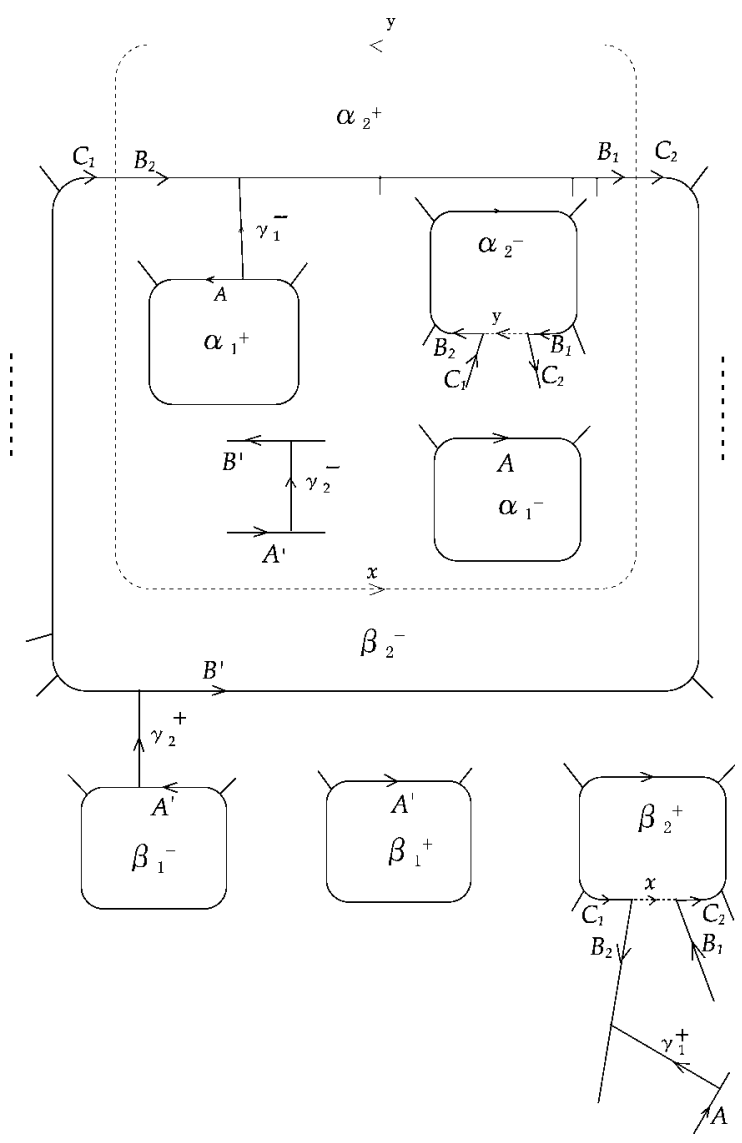

FIgURE 10. The DS-diagram $\Delta_{0}$ of the derived spine $P_{0}=\tilde{P}-\lambda_{0}$.

The codes $\phi\left(c_{i}\right), \phi\left(d_{i}\right)(2 \leq i \leq p-1)$ depend on the initial Heegaard diagram. As a consequence of the remodeling operation together with Lemma 4.1.1, we get the following lemma, whose proof is given in $\$ 4.5$.

LEMMA 4.2.1. Let $M$ be an oriented closed 3-manifold with $H G(M)=2$. Then there exists a DS-diagram with E-cycle $\Delta_{1}$ which satisfies $M=M\left(\Delta_{1}\right)$ and has a representation of the E-data:

$$
\begin{aligned}
\mathcal{E}\left(\Delta_{1}\right)= & \left\{a_{1}^{-\ell} d_{p}^{-r} c_{p}^{-\ell} a_{2}^{-r} a_{3}^{+r} a_{4}^{+\ell} a_{5}^{-\ell} a_{6}^{-r} a_{7}^{+r} a_{8}^{+\ell} a_{9}^{-\ell} a_{10}^{-r} a_{2}^{+r} a_{5}^{+\ell} a_{11}^{-\ell} a_{12}^{-r} \Lambda_{1}^{-}\right. \\
& a_{6}^{+r} a_{9}^{+\ell} a_{8}^{-\ell} a_{3}^{-r} a_{10}^{+r} c_{p}^{+\ell} \Upsilon_{1}^{+} c_{1}^{+r} b_{1}^{+r} b_{2}^{+r} b_{3}^{+r} d_{1}^{+\ell} \Upsilon_{2}^{+} d_{p}^{+r} a_{11}^{+\ell} a_{4}^{-\ell} a_{7}^{-r} a_{12}^{+r} a_{1}^{+\ell} \Lambda_{2}^{-} \\
& \Omega_{21}^{+} b_{4}^{-\ell} b_{5}^{-r} b_{6}^{+r} b_{2}^{-r} b_{7}^{+\ell} b_{8}^{-\ell} b_{9}^{-r} \Omega_{11}^{+} c_{1}^{-r} d_{1}^{-\ell} \Omega_{12}^{+} \\
& \left.b_{5}^{+r} b_{3}^{-r} b_{8}^{+\ell} b_{7}^{-\ell} b_{6}^{-r} b_{9}^{+r} b_{1}^{-r} b_{4}^{+\ell} \Omega_{22}^{+}\right\},
\end{aligned}
$$


where

(1) $\Upsilon_{1}^{+}=c_{p-1}^{+} c_{p-2}^{+} \cdots c_{2}^{+}$and $\Upsilon_{2}^{+}=d_{2}^{+} d_{3}^{+} \cdots d_{p-1}^{+}$,

(2) $\Lambda_{1}^{-}, \Lambda_{2}^{-}$are negative subwords and $\Omega_{11}^{+}, \Omega_{12}^{+}, \Omega_{21}^{+}$and $\Omega_{22}^{+}$are positive subwords,

(3) $\Lambda_{1}^{-} \cup \Lambda_{2}^{-}-\left(\cup c_{i}^{-} \cup \cup d_{i}^{-}\right), \Omega_{11}^{+}, \Omega_{12}^{+}, \Omega_{21}^{+}$and $\Omega_{22}^{+}$consist of the vertices inherited from the original Heegaard-diagram,

(4) $f\left(\Lambda_{1}^{-}\right) \cup f\left(\Lambda_{2}^{-}\right)=f\left(\Omega_{11}^{+}\right) \cup f\left(\Omega_{12}^{+}\right) \cup f\left(\Omega_{21}^{+}\right) \cup f\left(\Omega_{22}^{+}\right) \cup f\left(\Upsilon_{1}^{+}\right) \cup f\left(\Upsilon_{2}^{+}\right)$.

4.3. Some more moves. The E-data of Lemma 4.2.1 has $b l\left(\Delta_{1}\right)=14$. In order to prove the main theorem, we have to reduce the block number of $\Delta_{1}$. So we prepare several moves which are some compositions of regular moves and their inverses.

4.3.1. Exchange of vertices. Composing the regular moves $R_{1}^{ \pm 1}$ and $R_{2}^{ \pm 1}$, we can obtain eight moves $T_{\sigma_{2}, \sigma_{3}}^{\sigma_{1}}\left(\sigma_{j}=\ell\right.$ or $r$ ) (cf. [9]) which causes the following replacement of subwords in the coded sequence $\mathcal{E}(\Delta)$ :
$T_{\ell, \ell}^{\ell}: \quad$ (i) $b^{+\ell} a^{-\ell} \rightarrow a^{-\ell} b^{+\ell}$,
(ii) $a^{+\ell} \rightarrow a^{+\ell} x^{+\ell}$,
(iii) $b^{-\ell} \rightarrow x^{-\ell} b^{-\ell}$
$T_{\ell, r}^{\ell}: \quad$ (i) $b^{+r} a^{-\ell} \rightarrow a^{-\ell} b^{+r}$,
(ii) $a^{+\ell} \rightarrow a^{+\ell} x^{+r}$,
(iii) $b^{-r} \rightarrow b^{-r} x^{-r}$
$T_{r, \ell}^{\ell}:$ (i) $b^{+\ell} a^{-r} \rightarrow a^{-r} b^{+\ell}$,
(ii) $a^{+r} \rightarrow x^{+r} a^{+r}$,
(iii) $b^{-\ell} \rightarrow x^{-r} b^{-\ell}$
$T_{r, r}^{\ell}:$ (i) $b^{+r} a^{-r} \rightarrow a^{-r} b^{+r}$,
(ii) $a^{+r} \rightarrow x^{+\ell} a^{+r}$,
(iii) $b^{-r} \rightarrow b^{-r} x^{-\ell}$
$T_{\ell, \ell}^{r}: \quad$ (i) $a^{-\ell} b^{+\ell} \rightarrow b^{+\ell} a^{-\ell}$,
(ii) $a^{+\ell} \rightarrow a^{+\ell} x^{+r}$,
(iii) $b^{-\ell} \rightarrow x^{-r} b^{-\ell}$
$T_{\ell, r}^{r}:$ (i) $a^{-\ell} b^{+r} \rightarrow b^{+r} a^{-\ell}$,
(ii) $a^{+\ell} \rightarrow a^{+\ell} x^{+\ell}$,
(iii) $b^{-r} \rightarrow b^{-r} x^{-\ell}$
$T_{r, \ell}^{r}: \quad$ (i) $a^{-r} b^{+\ell} \rightarrow b^{+\ell} a^{-r}$,
(ii) $a^{+r} \rightarrow x^{+\ell} a^{+r}$,
(iii) $b^{-\ell} \rightarrow x^{-\ell} b^{-\ell}$
$T_{r, r}^{r}: \quad\left(\right.$ i) $a^{-r} b^{+r} \rightarrow b^{+r} a^{-r}$,
(ii) $a^{+r} \rightarrow x^{+r} a^{+r}$,
(iii) $b^{-r} \rightarrow b^{-r} x^{-r}$.

where $x^{ \pm \phi(x)}$ are new symbols appearing by each moves.

Each of the above eight moves $T_{\sigma_{2}, \sigma_{3}}^{\sigma_{1}}$ exchanges the positions of $a^{-\sigma_{2}}$ and $b^{+\sigma_{3}}$ in the coded sequence $\mathcal{E}(\Delta)$, and so we can use them for decreasing the block number without altering the regular equivalence class.

4.3.2. Block reducing lemma. By adequate successive applications of the moves $T_{\sigma, \sigma^{\prime}}^{\ell}$ or $T_{\sigma, \sigma^{\prime}}^{r}$, we have the following two lemmas. We call each of those lemmas a block reducing lemma.

LEMMA 4.3.1. Let $\Delta=(G, f, P ; e)$ be a DS-diagram with E-cycle having an arrangement of E-data of the form

$$
\mathcal{A}(\Delta)=W W_{0}^{-} U_{1}^{+} U_{2}^{-} \cdots U_{2 m-1}^{+} U_{2 m}^{-}
$$

where $W$ is a subword, $W_{0}^{-}$and $U_{2 k}^{-}$are negative blocks, $U_{2 k+1}^{+}$are positive blocks. If

$$
\left(\bigcup_{i=1}^{k} f\left(U_{2 i-1}^{+}\right)\right) \cap f\left(U_{2 k}^{-}\right)=\emptyset
$$


for any $k=1 \cdots m$, then we can obtain a DS-diagram $\Delta^{\prime}$ with E-cycle such that $\Delta^{\prime} \cong \Delta$ and $b l\left(\Delta^{\prime}\right)=b l(\Delta)-m$.

LEMMA 4.3.2. Let $\Delta=(G, f, P ; e)$ be a DS-diagram with E-cycle having an arrangement of E-data of the form

$$
\mathcal{A}(\Delta)=W W_{0}^{+} U_{1}^{-} U_{2}^{+} \cdots U_{2 m-1}^{-} U_{2 m}^{+}
$$

where $W$ is a subword, $W_{0}^{+}$and $U_{2 k}^{+}$are positive blocks, $U_{2 k+1}^{-}$are negative blocks. If

$$
\left(\bigcup_{i=1}^{k} f\left(U_{2 i-1}^{-}\right)\right) \cap f\left(U_{2 k}^{+}\right)=\emptyset
$$

for any $k=1 \cdots m$, then we can obtain a DS-diagram $\Delta^{\prime}$ with E-cycle such that $\Delta^{\prime} \cong \Delta$ and $\operatorname{rbl}\left(\Delta^{\prime}\right)=b l(\Delta)-m$.

These block reducing lemmas can be proved by applying the following lemmas 4.3.3 and 4.3.4 which were shown in [2] and give the moves exchanging the position of a positive subword and a negative subword in the arrangement $\mathcal{A}(\Delta)$.

LEMMA 4.3.3. Let $\Delta=(G, f, P ; e)$ be a DS-diagram having an arrangement of E-data of the form

$$
\mathcal{A}(\Delta)=U_{1}^{+} U_{2}^{-} W_{0}^{-} W_{1}^{+} W_{2}^{-} \cdots \cdots W_{2 n-1}^{+} W_{2 n}^{-} W_{2 n+1}^{+}
$$

where $U_{1}^{+}, W_{2 k-1}^{+}$are positive subwords and $U_{2}^{-}, W_{2 k}^{-}$are negative subwords.

If $f\left(U_{1}^{+}\right) \cap f\left(U_{2}^{-}\right)=\emptyset, U_{1}^{+}=b_{\nu}^{+} b_{\nu-1}^{+} \cdots b_{2}^{+} b_{1}^{+}$and $U_{2}^{-}=a_{1}^{-} a_{2}^{-} \cdots a_{\mu-1}^{-} a_{\mu}^{-}$, then, by adequate successive applications of the moves $T_{\sigma, \sigma^{\prime}}^{\ell}$, we can obtain a DS-diagram $\Delta^{\prime}$ such that $\Delta^{\prime} \cong \Delta$ and the E-data $\mathcal{E}\left(\Delta^{\prime}\right)$ has an arrangement

$$
\mathcal{A}\left(\Delta^{\prime}\right)=U_{2}^{-} U_{1}^{+} \tilde{W}_{0}^{-} \tilde{W}_{1}^{+} \tilde{W}_{2}^{-} \cdots \cdots \tilde{W}_{2 n}^{-} \tilde{W}_{2 n+1}^{+},
$$

satisfying the following conditions (i)-(iii):

(i) $\tilde{W}_{2 k}^{+}\left(\right.$or $\left.\tilde{W}_{2 k-1}^{-}\right)$is a positive (respectively negative) subword which differs from $W_{2 k}^{+}$(respectively $W_{2 k-1}^{-}$) only by the new symbols created by the moves,

(ii) $\tilde{W}_{2 k}^{+}$includes some new symbols if and only if the symbol $a_{i}^{+}$appears in the original subword $W_{2 k}^{+}$for some $i=1,2, \cdots, \mu$,

(iii) $\tilde{W}_{2 k-1}^{-}$includes some new symbols if and only if the original subword $W_{2 k-1}^{-}$contains the symbol $b_{j}^{-}$for some $j=1,2, \cdots, v$.

By similar successive applications of $T_{\sigma, \sigma^{\prime}}^{r}$ instead of $T_{\sigma, \sigma^{\prime}}^{\ell}$, we have that

LEMmA 4.3.4. Let $\Delta=(G, f, P ; e)$ be a DS-diagram having an arrangement of E-data of the form

$$
\mathcal{A}(\Delta)=U_{1}^{-} U_{2}^{+} W_{0}^{+} W_{1}^{-} W_{2}^{+} \cdots \cdots W_{2 n-1}^{-} W_{2 n}^{+} W_{2 n+1}^{-} .
$$


If $f\left(U_{1}^{-}\right) \cap f\left(U_{2}^{+}\right)=\emptyset, U_{1}^{-}=b_{v}^{-} b_{v-1}^{-} \cdots b_{2}^{-} b_{1}^{-}$and $U_{2}^{+}=a_{1}^{+} a_{2}^{+} \cdots a_{\mu-1}^{+} a_{\mu}^{+}$, then, by adequate successive applications of the moves $T_{\sigma, \sigma^{\prime}}^{r}$, we can obtain a DS-diagram $\Delta^{\prime}$ such that $\Delta^{\prime} \cong \Delta$ and the $E$-data $\mathcal{E}\left(\Delta^{\prime}\right)$ has an arrangement

$$
\mathcal{A}\left(\Delta^{\prime}\right)=U_{2}^{+} U_{1}^{-} \tilde{W}_{0}^{+} \tilde{W}_{1}^{-} \tilde{W}_{2}^{+} \cdots \cdots \tilde{W}_{2 n}^{+} \tilde{W}_{2 n+1}^{-},
$$

satisfying the following conditions (i)-(iii):

(i) $\tilde{W}_{2 k}^{-}$(or $\tilde{W}_{2 k-1}^{+}$) is a negative (respectively positive) subword which differs from $W_{2 k}^{-}$(respectively $W_{2 k-1}^{+}$) only by the new symbols created by the moves,

(ii) $\tilde{W}_{2 k}^{-}$includes some new symbols if and only if the symbol $a_{i}^{-}$appears in the original subword $W_{2 k}^{-}$for some $i=1,2, \cdots, \mu$,

(iii) $\tilde{W}_{2 k-1}^{+}$includes some new symbols if and only if the original subword $W_{2 k-1}^{+}$contains the symbol $b_{j}^{+}$for some $j=1,2, \cdots, v$.

In the case where the formula (4) is a blockwise representation, namely $W_{0}^{-}=W_{2 n+1}^{+}=$ $\emptyset$, the resulting arrangement (5) gives a blockwise representation

$$
\mathcal{A}\left(\Delta^{\prime}\right)=\left(U_{1}^{+} \tilde{W}_{1}^{+}\right) \tilde{W}_{2}^{-} \tilde{W}_{3}^{+} \cdots \cdots \tilde{W}_{2 n-1}^{+}\left(\tilde{W}_{2 n}^{-} U_{2}^{-}\right),
$$

and we have $b l\left(\Delta^{\prime}\right)=b l(\Delta)-1$.

Proof of Lemma 4.3.1. Let $\Delta=(G, f, P ; e)$ be a DS-diagram with E-cycle having an arrangement of E-data of the form

$$
\mathcal{A}(\Delta)=W W_{0}^{-} U_{1}^{+} U_{2}^{-} \cdots U_{2 m-1}^{+} U_{2 m}^{-}
$$

having the condition (2). Since $f\left(U_{1}^{+}\right) \cap f\left(U_{2}^{-}\right)=\emptyset$, we can apply Lemma 4.3.3 and get a regularly equivalent DS-diagram $\Delta_{1}=\left(G_{1}, f_{1}, P_{1} ; e_{1}\right)$ having an arrangement of E-data of the form

$$
\mathcal{A}\left(\Delta_{1}\right)=W_{1}\left(W_{1,0}^{-} U_{1,2}^{-}\right)\left(U_{1,1}^{+} U_{1,3}^{+}\right) U_{1,4}^{-} \cdots U_{1,2 m-1}^{+} U_{1,2 m}^{-}
$$

where each subword satisfies that

(1-i) $\quad W_{1}$ differs from $W$ only by new symbols $x_{1, j}^{ \pm}$produced by the moves,

(1-ii) $\quad W_{1,0}^{-}$differs from $W_{0}^{+}$only by new symbols $x_{1, j}^{-}$produced by the moves,

(1-iii) $U_{1,1}^{+}=U_{1}^{+}$and $U_{1,2}^{-}=U_{2}^{-}$

(1-iv) $U_{1,2 k-1}^{+}(k=2,3, \cdots m)$ differs from $U_{2 k-1}^{+}$only by new symbols $x_{1, j}^{+}$produced by the moves,

(1-v) $U_{1,2 k}^{-}(k=2,3, \cdots m)$ differs from $U_{2 k}^{-}$only by new symbols $x_{1, j}^{-}$produced by the moves. 
This implies that $b l\left(\Delta_{1}\right)=b l(\Delta)-1$. By the fact $f\left(U_{2 k}^{-}\right) \cap f\left(U_{2}^{-}\right)=\emptyset(k=2, \cdots, m)$ and the conditions (2), (1-iii)-(1-v), we can see the conditions

$$
\left(\bigcup_{i=1}^{k} f_{1}\left(U_{1,2 i-1}^{+}\right)\right) \cap f_{1}\left(U_{1,2 k}^{-}\right)=\emptyset
$$

for any $k=2,3, \cdots m$. Since $\left(f_{1}\left(U_{1,1}^{+}\right) \cup f_{1}\left(U_{1,3}^{+}\right)\right) \cap f_{1}\left(U_{1,4}^{-}\right)=\emptyset$, we can apply Lemma 4.3.3 and get a regularly equivalent DS-diagram $\Delta_{2}=\left(G_{2}, f_{2}, P_{2} ; e_{2}\right)$ having an arrangement of the form

$$
\mathcal{A}\left(\Delta_{2}\right)=W_{2}\left(W_{2,0}^{-} U_{2,2}^{-} U_{2,4}^{-}\right)\left(U_{2,1}^{+} U_{2,3}^{+} U_{2,5}^{+}\right) U_{2,6}^{-} \cdots U_{2,2 m-1}^{+} U_{2,2 m}^{-}
$$

where each subword satisfies that

(2-i) $\quad W_{2}$ differs from $W_{1}$ only by new symbols $x_{2, j}^{ \pm}$produced by the moves,

(2-ii) $W_{2,0}^{-}$differs from $W_{1}^{+}$only by new symbols $x_{2, j}^{-}$produced by the moves,

(2-iii) $\quad U_{2,2 k-1}^{+}=U_{1,2 k-1}^{+}(k=1,2)$ and $U_{2,2 k}^{-}=U_{1,2 k}^{-}(k=2)$

(2-iv) $U_{2,2 k-1}^{+}(k=3,4, \cdots m)$ differs from $U_{1,2 k-1}^{+}$only by new symbols $x_{2, j}^{+}$produced by the moves,

(2-v) $U_{2,2 k}^{-}(k=1,3,4, \cdots m)$ differs from $U_{1,2 k}^{-}$only by new symbols $x_{2, j}^{-}$produced by the moves.

This implies that $b l\left(\Delta_{2}\right)=b l(\Delta)-2$. By the fact $f\left(U_{2 k}^{-}\right) \cap f\left(U_{4}^{-}\right)=\emptyset(k=3, \cdots m)$ and the conditions (6), (2-iii)-(2-v), we can see the conditions that

$$
\left(\bigcup_{i=1}^{k} f\left(U_{2,2 i-1}^{+}\right)\right) \cap f\left(U_{2,2 k}^{-}\right)=\emptyset
$$

for any $k=3,4, \cdots m$.

Applying Lemma 4.3.3 $m$-times similarly, we get a sequence $\Delta_{k}=\left(G_{k}, f_{k}, P_{k} ; e_{k}\right)$ $(k=1,2, \cdots m)$ of regularly equivalent DS-diagrams, such that $\Delta_{k}$ is obtained from $\Delta_{k-1}$ by exchanging the positive subword $\left(U_{k-1,1}^{+} U_{k-1,3}^{+} \cdots U_{k-1,2 k-1}^{+}\right)$and the negative subwords $U_{k-1,2 k}^{-}$. By $x_{k, j}^{ \pm}\left(1 \leq j \leq s_{k}\right)$, we denote the new symbols created by the $k$-th application of Lemma 4.3.3 which deforms $\Delta_{k-1}$ into $\Delta_{k}$, where the number $s_{k}$ is given by

$$
s_{k}=\left(\# U_{2 k}^{-}\right) \times\left(\prod_{j=1}^{k}\left(\# U_{2 j-1}^{+}\right)\right) \text {. }
$$

The conditions (2) imply that the arrangement of $m$-th DS-diagram $\Delta_{m}$ can be represented as

$$
\begin{aligned}
\mathcal{A}\left(\Delta_{m}\right) & =W_{m} \Theta_{1}^{-} \Theta_{2}^{+} \\
& \left(\Theta_{1}^{-}=W_{m, 0}^{-} U_{m, 2}^{-} U_{m, 4}^{-} U_{m, 6}^{-} \cdots U_{m, 2 m}^{-}, \Theta_{2}^{+}=U_{m, 1}^{+} U_{m, 3}^{+} U_{m, 5}^{+} \cdots U_{m, 2 m-1}^{+}\right),
\end{aligned}
$$

and satisfies that 
(m-i) $\quad W_{m}$ differs from $W$ by some of new symbols $x_{k^{\prime}, j}^{ \pm}$for $k \leq k^{\prime} \leq m$,

(m-ii) $\quad W_{m, 0}^{-}$differs from $W_{0}^{-}$by some of new symbols $x_{k^{\prime}, j}^{-}$for $k \leq k^{\prime} \leq m$, and

(m-iii) $U_{m, 2 k-1}^{+}$differs from $U_{2 k-1}^{+}$by some of new symbols $x_{k^{\prime}, j}^{+}$for $k \leq k^{\prime} \leq m$, and

(m-iv) $U_{m, 2 k}^{-}$differs from $U_{2 k}^{-}$by new symbols $x_{k, j}^{-}$.

This implies that $b l\left(\Delta_{m}\right)=b l(\Delta)-m$. So we complete the proof by $\Delta_{m}=\Delta^{\prime}$.

Lemma 4.3.2 can be proved by using the moves in Lemma 4.3.4 instead of those in Lemma 4.3.3.

4.3.3. Pair eliminating. There are some cases where, even if the block reducing lemma is not directly applicable, we can reduce the block number after preparatory moves. Here we will introduce such preparatory moves, called pair eliminatings.

Consider the case that the given E-data includes subwords $W_{0}=a^{+\ell} W^{-} b^{+r}$ and $V_{0}=$ $a^{-\ell} b^{-r}$, where $W^{-}=w_{1}^{-\phi\left(w_{1}\right)} w_{2}^{-\phi\left(w_{2}\right)} \cdots w_{\zeta}^{-\phi\left(w_{\zeta}\right)}$ is a negative subword disjoint from $V_{0}$. Then, making successive $\zeta$ times applications of the moves $T_{\phi\left(w_{i}\right), \ell}^{\ell}(\sigma=\ell$ or $r$ ), we obtain an E-data in which the subwords $W_{0}, V_{0}$ and each of $w_{i}^{+\phi\left(w_{i}\right)}(i=1,2, \cdots \zeta)$ are replaced as following

$$
\begin{aligned}
& \text { (i) } a^{+\ell} W^{-} b^{+r} \rightarrow W^{-} a^{+\ell} b^{+r}, \quad \text { (ii) } a^{-\ell} b^{-r} \rightarrow V^{-} a^{-\ell} b^{-r}, \\
& \text { (iii) } w_{i}^{+\phi\left(w_{i}\right)} \rightarrow W_{i}^{+}(i=1,2, \cdots \zeta)
\end{aligned}
$$

where $V^{-}=v_{1}^{-\phi\left(v_{1}\right)} v_{2}^{-\phi\left(v_{2}\right)} \cdots v_{\zeta}^{-\phi\left(v_{\zeta}\right)}$ is a negative subword, and

$$
W_{i}^{+}= \begin{cases}w_{i}^{+\ell} v_{i}^{+\ell} & \text { if } \phi\left(w_{i}\right)=\ell \\ v_{i}^{+r} w_{i}^{+r} & \text { if } \phi\left(w_{i}\right)=r\end{cases}
$$

is a positive subword. The symbols above $v_{i}^{ \pm \phi\left(v_{i}\right)}(i=1,2, \cdots \zeta)$ are new symbols created by the moves $T_{\sigma, \ell}^{\ell}$.

Furthermore, making an application of the move $R_{2}^{+}$, we obtain an E-data in which the subwords $W^{-} a^{+\ell} b^{+r}, V^{-} a^{-\ell} b^{-r}$ are replaced by $W^{-}, V^{-}$respectively. Then, we obtain the move which causes the following replacement of subwords on the coded sequence

$$
\begin{gathered}
\mathfrak{S}_{+}^{\ell, r}: \text { (i) } a^{+\ell} W^{-} b^{+r} \rightarrow W^{-}, \quad \text { (ii) } a^{-\ell} b^{-r} \rightarrow V^{-}, \\
\quad \text { (iii) } w_{i}^{+\phi\left(w_{i}\right)} \rightarrow W_{i}^{+}(i=1,2, \cdots \zeta) .
\end{gathered}
$$

This move eliminates the vertices $a^{ \pm}$and $b^{ \pm}$in the arrangement $\mathcal{A}(\Delta)$. So we call $\mathfrak{S}_{+}^{\ell, r}$ a pair eliminating and a pair of vertices $a$ and $b$ an eliminating pair. 
Composing $T_{\sigma_{2}, \sigma_{3}}^{\sigma_{1}}$ and $R_{2}^{+}\left(\sigma_{j}=\ell\right.$ or $r$ ) similarly, we can obtain three other pair eliminatings $\mathfrak{S}_{+}^{r, \ell}, \mathfrak{S}_{-}^{\ell, r}$ and $\mathfrak{S}_{-}^{r, \ell}$, in a coded sequence:
$\mathfrak{S}_{+}^{r, \ell}:$
(i) $a^{+r} W^{-} b^{+\ell} \rightarrow W^{-}$,
(ii) $b^{-\ell} a^{-r} \rightarrow \bar{V}^{-}$,
(iii) $w_{i}^{+\phi\left(w_{i}\right)} \rightarrow{\overline{W_{i}}}^{+}(i=1,2, \cdots \zeta)$
$\mathfrak{S}_{-}^{\ell, r}: \quad$ (i) $a^{-\ell} W^{+} b^{-r} \rightarrow W^{+}$,
(ii) $b^{+r} a^{+\ell} \rightarrow \bar{V}^{+}$
(iii) $w_{i}^{-\phi\left(w_{i}\right)} \rightarrow{\overline{W_{i}}}^{-}(i=1,2, \cdots \zeta)$
$\mathfrak{S}_{-}^{r, \ell}: \quad$ (i) $a^{-r} W^{+} b^{-\ell} \rightarrow W^{+}$,
(ii) $a^{+r} b^{+\ell} \rightarrow V^{+}$,
(iii) $w_{i}^{-\phi\left(w_{i}\right)} \rightarrow W_{i}^{-}(i=1,2, \cdots \zeta)$

where

$$
\begin{aligned}
& W^{-}=w_{1}^{-\phi\left(w_{1}\right)} w_{2}^{-\phi\left(w_{2}\right)} \cdots w_{\zeta}^{-\phi\left(w_{\zeta}\right)}, \quad W^{+}=w_{1}^{+\phi\left(w_{1}\right)} w_{2}^{+\phi\left(w_{2}\right)} \cdots w_{\zeta}^{+\phi\left(w_{\zeta}\right)}, \\
& \bar{V}^{-}=v_{\zeta}^{-\phi\left(v_{\zeta}\right)} \cdots v_{2}^{-\phi\left(y_{2}\right)} v_{1}^{-\phi\left(v_{1}\right)}, \quad{\overline{W_{i}}}^{+}= \begin{cases}w_{i}^{+\ell} v_{i}^{+r} & \text { if } \phi\left(w_{i}\right)=\ell, \\
v_{i}^{+\ell} w_{i}^{+r} & \text { if } \phi\left(w_{i}\right)=r,\end{cases} \\
& \bar{V}^{+}=v_{\zeta}^{+\phi\left(v_{\zeta}\right)} \cdots v_{2}^{+\phi\left(y_{2}\right)} v_{1}^{+\phi\left(v_{1}\right)}, \quad{\overline{W_{i}}}^{-}= \begin{cases}w_{i}^{-r} v_{i}^{-\ell} & \text { if } \phi\left(w_{i}\right)=r, \\
v_{i}^{-r} w_{i}^{-\ell} & \text { if } \phi\left(w_{i}\right)=\ell,\end{cases} \\
& V^{+}=v_{1}^{+\phi\left(v_{1}\right)} v_{2}^{+\phi\left(v_{2}\right)} \cdots v_{\zeta}^{+\phi\left(v_{\zeta}\right)} \quad \text { and } \quad W_{i}^{-}= \begin{cases}w_{i}^{-r} v_{i}^{-r} & \text { if } \phi\left(w_{i}\right)=r \\
v_{i}^{-\ell} w_{i}^{-\ell} & \text { if } \phi\left(w_{i}\right)=\ell\end{cases}
\end{aligned}
$$

The pair eliminating is useful for removing vertices which prevent us from applying the block reducing lemma or other moves. A new DS-diagram $\Delta^{\prime}$ obtained by applying the pair eliminating operation to a DS-diagram $\Delta$ satisfies that $\Delta^{\prime} \cong \Delta$ and $b l\left(\Delta^{\prime}\right)=b l(\Delta)$ or $b l(\Delta)-1$ or $b l(\Delta)-2$.

4.4. Reducing the block number. In this subsection, we reduce a block number of $\Delta_{1}$ from fourteen to two by applying three times of pair eliminatings and two times of block reducing lemmas introduced in $\$ 4.3$. The coded sequences in the following moves are so complicated that we bracket the subwords where we apply a pair eliminating.

First we apply a pair eliminating $\mathfrak{S}_{+}^{\ell, r}$ to the E-data

$$
\begin{aligned}
\mathcal{E}\left(\Delta_{1}\right)= & \left\{a_{1}^{-\ell} d_{p}^{-r} c_{p}^{-\ell} a_{2}^{-r} a_{3}^{+r}\left(a_{4}^{+\ell} a_{5}^{-\ell} a_{6}^{-r} a_{7}^{+r}\right) a_{8}^{+\ell} a_{9}^{-\ell} a_{10}^{-r} a_{2}^{+r}\left(a_{5}^{+\ell}\right) a_{11}^{-\ell} a_{12}^{-r} \Lambda_{1}^{-}\right. \\
& \left(a_{6}^{+r}\right) a_{9}^{+\ell} a_{8}^{-\ell} a_{3}^{-r} a_{10}^{+r} c_{p}^{+\ell} \Upsilon_{1}^{+} c_{1}^{+r} b_{1}^{+r} b_{2}^{+r} b_{3}^{+r} d_{1}^{+\ell} \Upsilon_{2}^{+} d_{p}^{+r} a_{11}^{+\ell} \\
& \left(a_{4}^{-\ell} a_{7}^{-r}\right) a_{12}^{+r} a_{1}^{+\ell} \Lambda_{2}^{-} \Omega_{21}^{+} b_{4}^{-\ell} b_{5}^{-r} b_{6}^{+r} b_{2}^{-r} b_{7}^{+\ell} b_{8}^{-\ell} b_{9}^{-r} \Omega_{11}^{+} c_{1}^{-r} d_{1}^{-\ell} \Omega_{12}^{+} \\
& \left.b_{5}^{+r} b_{3}^{-r} b_{8}^{+\ell} b_{7}^{-\ell} b_{6}^{-r} b_{9}^{+r} b_{1}^{-r} b_{4}^{+\ell} \Omega_{22}^{+}\right\} .
\end{aligned}
$$

In order to explain how the pair eliminating is applied, we rewrite $\mathcal{E}\left(\Delta_{1}\right)$ by

$$
\mathcal{E}\left(\Delta_{1}\right)=U_{1} W_{0} U_{2} V_{0} U_{3}
$$


where

$$
\begin{aligned}
U_{1}= & a_{1}^{-\ell} d_{p}^{-r} c_{p}^{-\ell} a_{2}^{-r} a_{3}^{+r}, \\
W_{0}= & a_{4}^{+\ell}\left(a_{5}^{-\ell} a_{6}^{-r}\right) a_{7}^{+r}, \\
U_{2}= & a_{8}^{+\ell} a_{9}^{-\ell} a_{10}^{-r} a_{2}^{+r}\left(a_{5}^{+\ell}\right) a_{11}^{-\ell} a_{12}^{-r} \Lambda_{1}^{-}\left(a_{6}^{+r}\right) a_{9}^{+\ell} a_{8}^{-\ell} a_{3}^{-r} a_{10}^{+r} c_{p}^{+\ell} \\
& \Upsilon_{1}^{+} c_{1}^{+r} b_{1}^{+r} b_{2}^{+r} b_{3}^{+r} d_{1}^{+\ell} \Upsilon_{2}^{+} d_{p}^{+r} a_{11}^{+\ell}, \\
V_{0}= & a_{4}^{-\ell} a_{7}^{-r} \text { and } \\
U_{3}= & a_{12}^{+r} a_{1}^{+\ell} \Lambda_{2}^{-} \Omega_{21}^{+} b_{4}^{-\ell} b_{5}^{-r} b_{6}^{+r} b_{2}^{-r} b_{7}^{+\ell} b_{8}^{-\ell} b_{9}^{-r} \Omega_{11}^{+} c_{1}^{-r} d_{1}^{-\ell} \Omega_{12}^{+} \\
& b_{5}^{+r} b_{3}^{-r} b_{8}^{+\ell} b_{7}^{-\ell} b_{6}^{-r} b_{9}^{+r} b_{1}^{-r} b_{4}^{+\ell} \Omega_{22}^{+} .
\end{aligned}
$$

Applying the pair eliminating, the subwords $W_{0}, V_{0}$ and the vertices $a_{5}^{+\ell}$ and $a_{6}^{+r}$ are replaced as following

$$
\begin{aligned}
& \text { (i) } a_{4}^{+\ell} W^{-} a_{7}^{+r} \rightarrow W^{-}=a_{5}^{-\ell} a_{6}^{-r} \text {, } \\
& \text { (ii) } a_{4}^{-\ell} a_{7}^{-r} \rightarrow V^{+}=m_{1}^{-\ell} m_{2}^{-r} \text {, } \\
& \text { (iii) } \begin{aligned}
a_{5}^{+\ell} \rightarrow W_{1}^{+}=a_{5}^{+\ell} m_{1}^{+\ell} \\
a_{6}^{+r} \rightarrow W_{2}^{+}=m_{2}^{+r} a_{6}^{+r} .
\end{aligned}
\end{aligned}
$$

As a consequence, we obtain a DS-diagram $\Delta_{2}$ with an E-data

$$
\begin{aligned}
\mathcal{E}\left(\Delta_{2}\right)= & U_{1} W^{-} \widehat{U_{2}} V^{-} U_{3} \\
= & \left\{a_{1}^{-\ell} d_{p}^{-r} c_{p}^{-\ell} a_{2}^{-r}\left(a_{3}^{+r}\left(a_{5}^{-\ell} a_{6}^{-r}\right) a_{8}^{+\ell}\right) a_{9}^{-\ell} a_{10}^{-r} a_{2}^{+r}\left(a_{5}^{+\ell} m_{1}^{+\ell}\right) a_{11}^{-\ell} a_{12}^{-r} \Lambda_{1}^{-}\right. \\
& \left(m_{2}^{+r} a_{6}^{+r}\right) a_{9}^{+\ell}\left(a_{8}^{-\ell} a_{3}^{-r}\right) a_{10}^{+r} c_{p}^{+\ell} \Upsilon_{1}^{+} c_{1}^{+r} b_{1}^{+r} b_{2}^{+r} b_{3}^{+r} d_{1}^{+\ell} r_{2}^{+} d_{p}^{+r} a_{11}^{+\ell} \\
& \left(m_{1}^{-\ell} m_{2}^{-r}\right) a_{12}^{+r} a_{1}^{+\ell} \Lambda_{2}^{-} \Omega_{21}^{+} b_{4}^{-\ell} b_{5}^{-r} b_{6}^{+r} b_{2}^{-r} b_{7}^{+\ell} b_{8}^{-\ell} b_{9}^{-r} \Omega_{11}^{+} c_{1}^{-r} d_{1}^{-\ell} \Omega_{12}^{+} \\
& \left.b_{5}^{+r} b_{3}^{-r} b_{8}^{+\ell} b_{7}^{-\ell} b_{6}^{-r} b_{9}^{+r} b_{1}^{-r} b_{4}^{+\ell} \Omega_{22}^{+}\right\} .
\end{aligned}
$$

The block number of $\Delta_{2}$ is still fourteen. This move from $\Delta_{1}$ to $\Delta_{2}$ enables us to make the following move.

Applying the pair eliminating $\mathfrak{S}_{+}^{r, \ell}$ to $\mathcal{E}\left(\Delta_{2}\right)$, which causes the replacement

(i) $a_{3}^{+r} W^{-} a_{8}^{+\ell} \rightarrow W^{-}=a_{5}^{-\ell} a_{6}^{-r}$,

(ii) $a_{8}^{-\ell} a_{3}^{-r} \rightarrow \bar{V}^{-}=m_{4}^{-\ell} m_{3}^{-r}$,

$$
\text { (iii) } \begin{aligned}
a_{5}^{+\ell} & \rightarrow a_{5}^{+\ell} m_{3}^{+r} \\
a_{6}^{+r} & \rightarrow m_{4}^{+\ell} a_{6}^{+r}
\end{aligned}
$$

we obtain a DS-diagram $\Delta_{3}$ with an E-data

$$
\begin{aligned}
\mathcal{E}\left(\Delta_{3}\right)= & \left\{a_{1}^{-\ell} d_{p}^{-r} c_{p}^{-\ell} a_{2}^{-r}\left(a_{5}^{-\ell} a_{6}^{-r}\right) a_{9}^{-\ell} a_{10}^{-r} a_{2}^{+r}\left(a_{5}^{+\ell} m_{3}^{+r} m_{1}^{+\ell}\right) a_{11}^{-\ell} a_{12}^{-r} \Lambda_{1}^{-}\right. \\
& \left(m_{2}^{+r} m_{4}^{+\ell} a_{6}^{+r}\right) a_{9}^{+\ell}\left(m_{4}^{-\ell} m_{3}^{-r}\right) a_{10}^{+r} c_{p}^{+\ell} \Upsilon_{1}^{+} c_{1}^{+r} b_{1}^{+r} b_{2}^{+r} b_{3}^{+r} d_{1}^{+\ell} \Upsilon_{2}^{+} d_{p}^{+r} a_{11}^{+\ell} \\
& \left(m_{1}^{-\ell} m_{2}^{-r}\right) a_{12}^{+r} a_{1}^{+\ell} \Lambda_{2}^{-} \Omega_{21}^{+} b_{4}^{-\ell} b_{5}^{-r} b_{6}^{+r} b_{2}^{-r} b_{7}^{+\ell} b_{8}^{-\ell} b_{9}^{-r} \Omega_{11}^{+} c_{1}^{-r} d_{1}^{-\ell} \Omega_{12}^{+} \\
& \left.b_{5}^{+r} b_{3}^{-r} b_{8}^{+\ell} b_{7}^{-\ell} b_{6}^{-r} b_{9}^{+r} b_{1}^{-r} b_{4}^{+\ell} \Omega_{22}^{+}\right\} .
\end{aligned}
$$


This move reduces the block number by two, that is, $b l\left(\Delta_{3}\right)=b l\left(\Delta_{2}\right)-2=12$.

Applying the pair eliminating $\mathfrak{S}_{+}^{r, \ell}$ to

$$
\begin{aligned}
\mathcal{E}\left(\Delta_{3}\right)= & \left\{a_{1}^{-\ell} d_{p}^{-r} c_{p}^{-\ell} a_{2}^{-r} a_{5}^{-\ell} a_{6}^{-r} a_{9}^{-\ell} a_{10}^{-r} a_{2}^{+r} a_{5}^{+\ell} m_{3}^{+r} m_{1}^{+\ell} a_{11}^{-\ell} a_{12}^{-r} \Lambda_{1}^{-}\right. \\
& m_{2}^{+r} m_{4}^{+\ell} a_{6}^{+r} a_{9}^{+\ell} m_{4}^{-\ell} m_{3}^{-r} a_{10}^{+r} c_{p}^{+\ell} \Upsilon_{1}^{+} c_{1}^{+r} b_{1}^{+r}\left(b_{2}^{+r}\right) b_{3}^{+r} d_{1}^{+\ell} \Upsilon_{2}^{+} d_{p}^{+r} a_{11}^{+\ell} \\
& m_{1}^{-\ell} m_{2}^{-r} a_{12}^{+r} a_{1}^{+\ell} \Lambda_{2}^{-} \Omega_{21}^{+} b_{4}^{-\ell} b_{5}^{-r}\left(b_{6}^{+r} b_{2}^{-r} b_{7}^{+\ell}\right) b_{8}^{-\ell} b_{9}^{-r} \Omega_{11}^{+} c_{1}^{-r} d_{1}^{-\ell} \Omega_{12}^{+} \\
& \left.b_{5}^{+r} b_{3}^{-r} b_{8}^{+\ell}\left(b_{7}^{-\ell} b_{6}^{-r}\right) b_{9}^{+r} b_{1}^{-r} b_{4}^{+\ell} \Omega_{22}^{+}\right\},
\end{aligned}
$$

which causes the replacement
(i) $b_{6}^{+r} W^{-} b_{7}^{+\ell} \rightarrow W^{-}=b_{2}^{-r}$,
(ii) $b_{7}^{-\ell} b_{6}^{-r} \rightarrow \bar{V}^{-}=m_{5}^{-\ell}$,
(iii) $b_{2}^{+r} \rightarrow m_{5}^{+\ell} b_{2}^{+r}$,

we obtain $\Delta_{4}$ with an E-data

$$
\begin{aligned}
\mathcal{E}\left(\Delta_{4}\right)= & \left\{a_{1}^{-\ell} d_{p}^{-r} c_{p}^{-\ell} a_{2}^{-r} a_{5}^{-\ell} a_{6}^{-r} a_{9}^{-\ell} a_{10}^{-r} a_{2}^{+r} a_{5}^{+\ell} m_{3}^{+r} m_{1}^{+\ell} a_{11}^{-\ell} a_{12}^{-r} \Lambda_{1}^{-}\right. \\
& m_{2}^{+r} m_{4}^{+\ell} a_{6}^{+r} a_{9}^{+\ell} m_{4}^{-\ell} m_{3}{ }^{-r} a_{10}^{+r} c_{p}^{+\ell} \Upsilon_{1}^{+} c_{1}^{+r} b_{1}^{+r}\left(m_{5}^{+\ell} b_{2}^{+r}\right) b_{3}^{+r} d_{1}^{+\ell} \Upsilon_{2}^{+} d_{p}^{+r} a_{11}^{+\ell} \\
& m_{1}^{-\ell} m_{2}{ }^{-r} a_{12}^{+r} a_{1}^{+\ell} \Lambda_{2}^{-} \Omega_{21}^{+} b_{4}^{-\ell} b_{5}^{-r}\left(b_{2}^{-r}\right) b_{8}^{-\ell} b_{9}^{-r} \Omega_{11}^{+} c_{1}^{-r} d_{1}^{-\ell} \Omega_{12}^{+} \\
& \left.b_{5}^{+r} b_{3}^{-r} b_{8}^{+\ell}\left(m_{5}^{-\ell}\right) b_{9}^{+r} b_{1}^{-r} b_{4}^{+\ell} \Omega_{22}^{+}\right\} .
\end{aligned}
$$

This move causes $b l\left(\Delta_{4}\right)=b l\left(\Delta_{3}\right)-2=10$.

Now we apply the block reducing lemma. In order to confirm the conditions for the block reducing lemma, we rewrite the E-data $\mathcal{E}\left(\Delta_{4}\right)$ by a blockwise representation:

$$
\begin{aligned}
\mathcal{E}\left(\Delta_{4}\right)= & W_{4,1}^{-} W_{4,2}^{+} W_{4,3}^{-} W_{4,4}^{+} W_{4,5}^{-} W_{4,6}^{+} W_{4,7}^{-} W_{4,8}^{+} W_{4,9}^{-} W_{4,10}^{+} \\
& W_{4,11}^{-} W_{4,12}^{+} W_{4,13}^{-} W_{4,14}^{+} W_{4,15}^{-} W_{4,16}^{+} W_{4,17}^{-} W_{4,18}^{+} W_{4,19}^{-} W_{4,20}^{+}
\end{aligned}
$$

where

$$
\begin{array}{llrl}
W_{4,1}^{-}=a_{1}^{-\ell} d_{p}^{-r} c_{p}^{-\ell} a_{2}^{-r} a_{5}^{-\ell} a_{6}^{-r} a_{9}^{-\ell} a_{10}^{-r} & W_{4,2}^{+}=a_{2}^{+r} a_{5}^{+\ell} m_{3}^{+r} m_{1}^{+\ell} \\
W_{4,3}^{-}=a_{11}^{-\ell} a_{12}^{-r} \Lambda_{1}^{-} & W_{4,4}^{+}=m_{2}^{+r} m_{4}^{+\ell} a_{6}^{+r} a_{9}^{+\ell} \\
W_{4,5}^{-}=m_{4}^{-\ell} m_{3}^{-r} & W_{4,6}^{+}=a_{10}^{+r} c_{p}^{+\ell} r_{1}^{+} c_{1}^{+r} b_{1}^{+r} m_{5}^{+\ell} b_{2}^{+r} b_{3}^{+r} d_{1}^{+\ell} r_{2}^{+} \\
W_{4,7}^{-}=m_{1}^{-\ell} m_{2}^{-r} & d_{p}^{+r} a_{11}^{+\ell} \\
W_{4,9}^{-}=\Lambda_{2}^{-} & W_{4,8}^{+}=a_{12}^{+r} a_{1}^{+\ell} \\
W_{4,11}^{-}=b_{4}^{-\ell} b_{5}^{-r} b_{2}^{-r} b_{8}^{-\ell} b_{9}^{-r} & W_{4,10}^{+}=\Omega_{21}^{+} \\
W_{4,13}^{-}=c_{1}^{-r} d_{1}^{-\ell} & W_{4,12}^{+}=\Omega_{11}^{+} \\
W_{4,15}^{-}=b_{3}^{-r} & W_{4,14}^{+}=\Omega_{12}^{+} b_{5}^{+r} \\
W_{4,17}^{-}=\left(m_{5}^{-\ell}\right) & W_{4,16}^{+}=b_{8}^{+\ell} \\
W_{4,19}^{-}=b_{1}^{-r} & W_{4,18}^{+}=b_{9}^{+r} \\
& W_{4,20}^{+}=b_{4}^{+\ell} \Omega_{22}^{+} .
\end{array}
$$

Obviously, $f\left(W_{4,5}^{-}\right) \cap f\left(W_{4,6}^{+}\right)=\emptyset$ and $\left\{f\left(W_{4,5}^{-}\right) \cup f\left(W_{4,7}^{-}\right\} \cap f\left(W_{4,8}^{+}\right)=\emptyset\right.$. We can apply Lemma 4.3.2 to the blocks $W_{4,5}^{-} W_{4,6}^{+} W_{4,7}^{-} W_{4,8}^{+}$in $\mathcal{E}\left(\Delta_{4}\right)$. Then we obtain a DS-diagram $\Delta_{5}$ 
which satisfies $b l\left(\Delta_{5}\right)=b l\left(\Delta_{4}\right)-2$. The E-data of $\Delta_{5}$ can be represented as

$$
\begin{aligned}
\mathcal{E}\left(\Delta_{5}\right)= & W_{5,1}^{-} W_{5,2}^{+} W_{5,3}^{-} W_{5,4}^{+} W_{5,9}^{-} W_{5,10}^{+} W_{5,11}^{-} W_{5,12}^{+} \\
& W_{5,13}^{-} W_{5,14}^{+} W_{5,15}^{-} W_{5,16}^{+} W_{5,17}^{-} W_{5,18}^{+} W_{5,19}^{-} W_{5,20}^{+}
\end{aligned}
$$

where

$$
\begin{array}{ll}
W_{5,1}^{-}=\tilde{W}_{4,1}^{-} & W_{5,2}^{+}=\tilde{W}_{4,2}^{+} \\
W_{5,3}^{-}=\tilde{W}_{4,3}^{-} & W_{5,4}^{+}=\tilde{W}_{4,4}^{+} W_{4,6}^{+} W_{4,8}^{+} \\
W_{5,9}^{-}=W_{4,5}^{-} W_{4,7}^{-} \tilde{W}_{4,9}^{-} & W_{5,10}^{+}=W_{4,10}^{+} \\
W_{5,11}^{-}=\tilde{W}_{4,11}^{-} & W_{5,12}^{+}=W_{4,12}^{+} \\
W_{5,13}^{-}=\tilde{W}_{4,13}^{-} & W_{5,14}^{+}=W_{4,14}^{+} \\
W_{5,15}^{-}=\tilde{W}_{4,15}^{-} & W_{5,16}^{+}=W_{4,16}^{+} \\
W_{5,17}^{-}=\tilde{W}_{4,17}^{-} & W_{5,18}^{+}=W_{4,18}^{+} \\
W_{5,19}^{-}=\tilde{W}_{4,19}^{-} & W_{5,20}^{+}=W_{4,20}^{+} .
\end{array}
$$

A subword $\tilde{W}_{4, i}^{-}$(or $\tilde{W}_{4, j}^{+}$) differs from $W_{4, i}^{-}$(or $W_{4, j}^{+}$) only by the new vertices created by the moves from $\Delta_{4}$ to $\Delta_{5}$. The fact $W_{5, j}^{+}=W_{4, j}^{+}(j=10,12,14,16,18$ or 20$)$ implies that

$$
\begin{aligned}
& f\left(W_{5,10}^{+}\right) \cap f\left(W_{5,11}^{-}\right)=\emptyset, \\
& \left\{f\left(W_{5,10}^{+}\right) \cup f\left(W_{5,12}^{+}\right)\right\} \cap f\left(W_{5,13}^{-}\right)=\emptyset, \\
& \left\{f\left(W_{5,10}^{+}\right) \cup f\left(W_{5,12}^{+}\right) \cup f\left(W_{5,14}^{+}\right)\right\} \cap f\left(W_{5,15}^{-}\right)=\emptyset, \\
& \left\{f\left(W_{5,10}^{+}\right) \cup f\left(W_{5,12}^{+}\right) \cup f\left(W_{5,14}^{+}\right) \cup f\left(W_{5,16}^{+}\right)\right\} \cap f\left(W_{5,17}^{-}\right)=\emptyset, \\
& \left\{f\left(W_{5,10}^{+}\right) \cup f\left(W_{5,12}^{+}\right) \cup f\left(W_{5,14}^{+}\right) \cup f\left(W_{5,16}^{+}\right) \cup f\left(W_{5,18}^{+}\right)\right\} \cap f\left(W_{5,19}^{-}\right)=\emptyset \quad \text { and } \\
& \left\{f\left(W_{5,10}^{+}\right) \cup f\left(W_{5,12}^{+}\right) \cup f\left(W_{5,14}^{+}\right) \cup f\left(W_{5,16}^{+}\right) \cup f\left(W_{5,18}^{+}\right) \cup f\left(W_{5,20}^{+}\right)\right\} \cap f\left(W_{5,1}^{-}\right)=\emptyset .
\end{aligned}
$$

Therefore applying Lemma 4.3.1, we obtain a DS-diagram $\Delta$ which has $b l(\Delta)=2$. This shows that it is sufficient for the proof of our main theorem to prove Lemma 4.2.1.

\subsection{Proofs of Lemma 4.1.1 and Lemma 4.2.1}

4.5.1. Proof of Lemma 4.1.1. According to [11], for a fake surface $\tilde{P}$ induced from a Heegaard diagram $\left(M_{1}, M_{2} ; \vec{D}_{1}, \vec{D}_{2}\right)$ with genus 2 , we can find two faces $\lambda_{1}$ and $\lambda_{1}^{\prime}$ on $\tilde{P}$ having the following conditions

(1) two faces $\lambda_{1}$ and $\lambda_{1}^{\prime}$ are included in $F \subset \tilde{P}$,

(2) there are five edges $A, B, D, A^{\prime}$ and $B^{\prime}$ such that

$$
\begin{array}{lll}
A \subset \tilde{h}\left(\partial \lambda_{1}^{-} \cap \partial \alpha_{1}^{+}\right), & B \subset \tilde{h}\left(\partial \lambda_{1}^{-} \cap \partial \alpha_{2}^{+}\right), & D \subset \tilde{h}\left(\partial \lambda_{1}^{+} \cap \partial \beta_{2}^{+}\right), \\
A^{\prime} \subset \tilde{h}\left(\partial \lambda_{1}^{\prime+} \cap \partial \beta_{1}^{-}\right) & \text {and } \quad & B^{\prime} \subset \tilde{h}\left(\partial \lambda_{1}^{\prime+} \cap \partial \beta_{2}^{-}\right),
\end{array}
$$

(3) these edges are arranged on $\partial \lambda_{1}$ and $\partial \lambda_{1}^{\prime}$ in the order

$$
\partial \lambda_{1}=\cdots A \cdots D B \cdots \quad \text { and } \quad \partial \lambda_{1}^{\prime}=\cdots A^{\prime} \cdots B^{\prime} \cdots
$$


Each symbols $\lambda_{1}^{ \pm}, \lambda_{1}^{\prime \pm}, \alpha_{i}^{ \pm}$and $\beta_{i}^{ \pm}$means each component of the inverse images $\tilde{h}^{-1}\left(\lambda_{1}\right)$, $\tilde{h}^{-1}\left(\lambda_{1}^{\prime}\right), \tilde{h}^{-1}\left(\alpha_{i}\right)$ and $\tilde{h}^{-1}\left(\beta_{i}\right)$ respectively, such that $\lambda_{1}^{-}, \lambda_{1}^{\prime-}$ and $\alpha_{i}^{ \pm}$are on $\partial \mathbf{B}_{1}^{3}$, and $\lambda_{1}^{+}$, $\lambda_{1}^{\prime+}$ and $\beta_{i}^{ \pm}$are on $\partial \mathbf{B}_{2}^{3}$.

Let $p \in A, q \in B, p^{\prime} \in A^{\prime}$ and $q^{\prime} \in B^{\prime}$ be four points. Then there are two simple arcs $\gamma_{1}$ on $\lambda_{1}$ and $\gamma_{2}$ on $\lambda_{1}^{\prime}$ which satisfies that

(1) $\gamma_{1}$ is a directed arc on $\lambda_{1}$ going from $p$ to $q$ and $\gamma_{2}$ is a directed arc on $\lambda_{1}^{\prime}$ going from $p^{\prime}$ to $q^{\prime}$,

(2) $\gamma_{1} \cap \gamma_{2}=\emptyset$ on $F$,

(3) $\tilde{h}^{-}\left(\gamma_{1}\right)$ has two components $\gamma_{1}^{+} \subset \lambda_{1}^{+}$and $\gamma_{1}^{-} \subset \lambda_{1}^{-}$,

(4) $\tilde{h}^{-}\left(\gamma_{2}\right)$ has two components $\gamma_{2}^{+} \subset \lambda_{1}^{\prime+}$ and $\gamma_{2}^{-} \subset \lambda_{1}^{\prime-}$.

The $\operatorname{arcs} \gamma_{1}^{ \pm}$and $\gamma_{2}^{ \pm}$on $\partial \mathbf{B}_{1}^{3} \cup \partial \mathbf{B}_{2}^{3}$ are as described in Figure 11 .

For a Heegaard diagram $\left(M_{1}, M_{2} ; \vec{D}_{1}, \vec{D}_{2}\right)$ with the arcs $\gamma_{1}$ and $\gamma_{2}$ on $\tilde{P}$, we apply a disk-slide to the meridian disks $\alpha_{2}$ and $\beta_{2}$ so that the edges $B$ and $C$ cross each other and a face $\lambda_{0}$ is produced, as described in Figure 12. This Heegaard diagram gives a fake surface admitting a 2-gon $\lambda_{0}$ and pre mark-lines $\gamma_{1}^{-}$and $\gamma_{2}^{+}$. This complete the proof.

4.5.2. Proof of Lemma 4.2.1. The lemma will be proved by carrying out the algorithm in [7]. First we briefly explain some terminologies, see [7] for detail. For each edge (or face) $\sigma$ of $\Delta$, we call $\sigma$ a 1-cell (or a 2-cell) of $\Delta$, we may call $f(\sigma)$ in $P$ a 1-label (or a 2-label, respectively) of $\Delta$. Let $\Delta=(G, f, P)$ be a general DS-diagram. Consider a pair of 2-cells in $\Delta$ with the same label $\alpha$. We denote one of them by $\alpha^{+}$and the other by $\alpha^{-}$. In this way, we can separate whole 2-cells in $\Delta$ into two classes $\left\{\alpha_{1}^{+}, \alpha_{2}^{+}, \cdots, \alpha_{n+1}^{+}\right\}$and $\left\{\alpha_{1}^{-}, \alpha_{2}^{-}, \cdots, \alpha_{n+1}^{-}\right\}$. The closure $\mathcal{Z}^{+}$of $\alpha_{1}^{+} \cup \alpha_{2}^{+} \cup \cdots \cup \alpha_{n+1}^{+}$(or $\mathcal{Z}^{-}$of $\alpha_{1}^{-} \cup \alpha_{2}^{-} \cup \cdots \cup \alpha_{n+1}^{-}$) is called the positive zone (or the negative zone, respectively). We will call such a pair $\left(\mathcal{Z}^{+}, \mathcal{Z}^{-}\right)$a bicoloring of the DS-diagram $\Delta$. If both of $\mathcal{Z}^{+}$and $\mathcal{Z}^{-}$are connected, we will call $\left(\mathcal{Z}^{+}, \mathcal{Z}^{-}\right)$a split bicoloring of $\Delta$. A DS-diagram $\Delta$ is splittable if $\Delta$ has a split bicoloring.

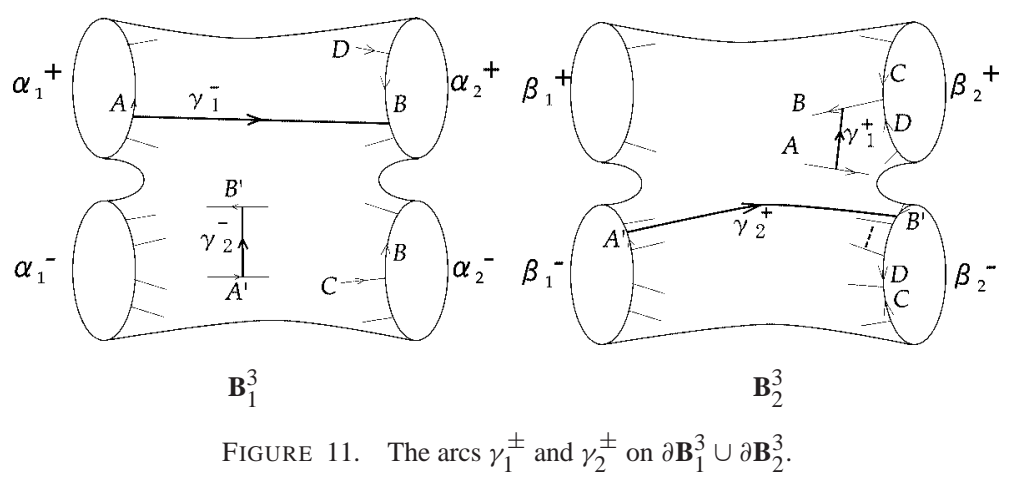




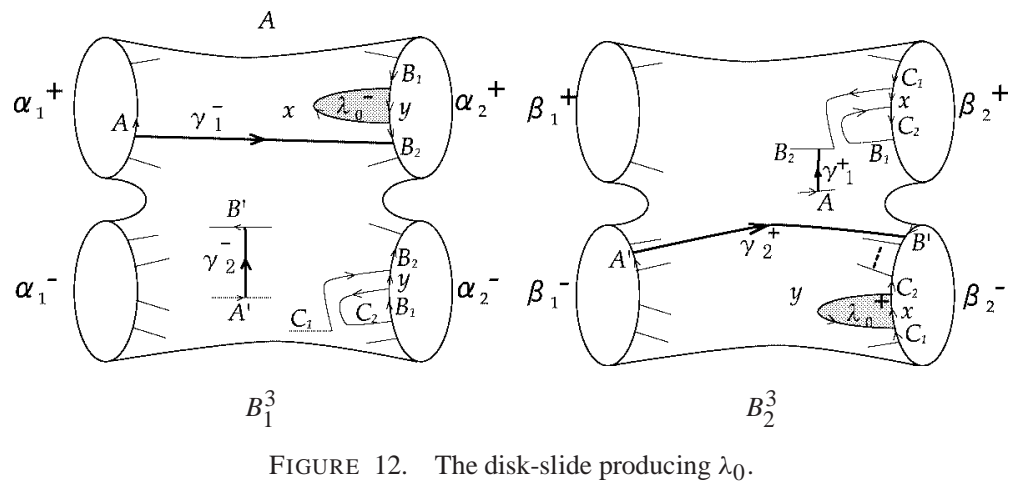

Let $\Delta$ be a splittable DS-diagram with a split bicoloring $\left(\mathcal{Z}^{+}, \mathcal{Z}^{-}\right)$. Let $a_{1}, a_{2}, \cdots, a_{m}$ be a sequence of successive 1-cells on a simple loop $\mathcal{Z}^{+} \cap \mathcal{Z}^{-}$such that $\operatorname{cl}\left(a_{1} \cup a_{2} \cup \cdots \cup a_{m}\right)=$ $\mathcal{Z}^{+} \cap \mathcal{Z}^{-}$. Let $A_{i}$ be the label of $a_{i}, 1 \leq i \leq m$. Then we say that $\Gamma=A_{1} A_{2} \cdots A_{m}$ is a splitting cycle of $\Delta$ associated with $\left(\mathcal{Z}^{+}, \mathcal{Z}^{-}\right)$. If a DS-diagram $\Delta$ has a splitting cycle $\Gamma=A_{1} A_{2} \cdots A_{m}$ satisfying $A_{i} \neq A_{j}$ for each $i \neq j$, then $\mathcal{Z}^{+} \cap \mathcal{Z}^{-}$is an E-cycle of $\Delta$.

For remodeling a DS-diagram into one with E-cycle, we use elementary deformations $I^{ \pm}, I I^{ \pm}$and digging operations as deformations of DS-diagrams which preserve the represented manifold, see [7].

Now we apply the remodeling algorithm to the DS-diagram $\Delta_{0}$ of the derived spine $P_{0}$ obtained by Lemma 4.1.1. We decide a bicoloring $\left(\mathcal{Z}^{+}, \mathcal{Z}^{-}\right)$of $\Delta_{0}$ by $Z^{+}=\left\{\alpha_{i}^{+}, \beta_{i}^{+}, \lambda_{j}^{+}\right\}$ and $Z^{-}=\left\{\alpha_{i}^{-}, \beta_{i}^{-}, \lambda_{j}^{-}\right\}$. The choice of $\lambda_{0}$ makes it possible to define this bicoloring. The intersection $\mathcal{Z}^{+} \cap \mathcal{Z}^{-}$consists of three cycles, and so this bicoloring $\left(\mathcal{Z}^{+}, \mathcal{Z}^{-}\right)$is not yet a split one. The three cycles of $\mathcal{Z}^{+} \cap \mathcal{Z}^{-}$can be written as

$$
\begin{aligned}
\partial \alpha_{1}^{+} & =\left\{A \Gamma\left(\alpha_{1}^{+}\right)\right\}, \\
\partial \beta_{1}^{-} & =\left\{\left(A^{\prime}\right)^{-1} \Gamma\left(\beta_{1}^{-}\right)\right\} \text {and } \\
\left(\partial \alpha_{2}^{+}-y\right) \cup\left(\partial \beta_{2}^{-}-x\right) & =\left\{B \Gamma\left(\alpha_{2}^{+}\right) C \Gamma\left(\beta_{21}^{-}\right)\left(B^{\prime}\right)^{-1} \Gamma\left(\beta_{22}^{-}\right)\right\},
\end{aligned}
$$

where $\Gamma\left(\alpha_{1}^{+}\right), \Gamma\left(\alpha_{2}^{+}\right), \Gamma\left(\beta_{1}^{-}\right), \Gamma\left(\beta_{21}^{-}\right)$and $\Gamma\left(\beta_{22}^{-}\right)$are consecutive sequences of 1 -labels, see Figure 13.

We apply DS-deformations in Theorem 2.3 of [7] and digging operations to make $Z^{+} \cap$ $Z^{-}$a simple loop. The DS-deformaions in Theorem 2.3 of [7] are applied to the 1-labels $B$ and $B^{\prime}$. As a consequence of these deformations, the three cycles of $\mathcal{Z}^{+} \cap \mathcal{Z}^{-}$are deformed into

$$
\begin{aligned}
\partial \alpha_{1}^{+} & =\left\{A \Gamma\left(\alpha_{1}^{+}\right)\right\}, \\
\partial \beta_{1}^{-} & =\left\{\left(A^{\prime}\right)^{-1} \Gamma\left(\beta_{1}^{-}\right)\right\} \quad \text { and } \\
\left(\partial \alpha_{2}^{+}-y\right) \cup\left(\partial \beta_{2}^{-}-x\right) & =\left\{\Gamma(B) \Gamma\left(\alpha_{2}^{+}\right) C \Gamma\left(\beta_{21}^{-}\right) \Gamma\left(B^{\prime}\right) \Gamma\left(\beta_{22}^{-}\right)\right\},
\end{aligned}
$$




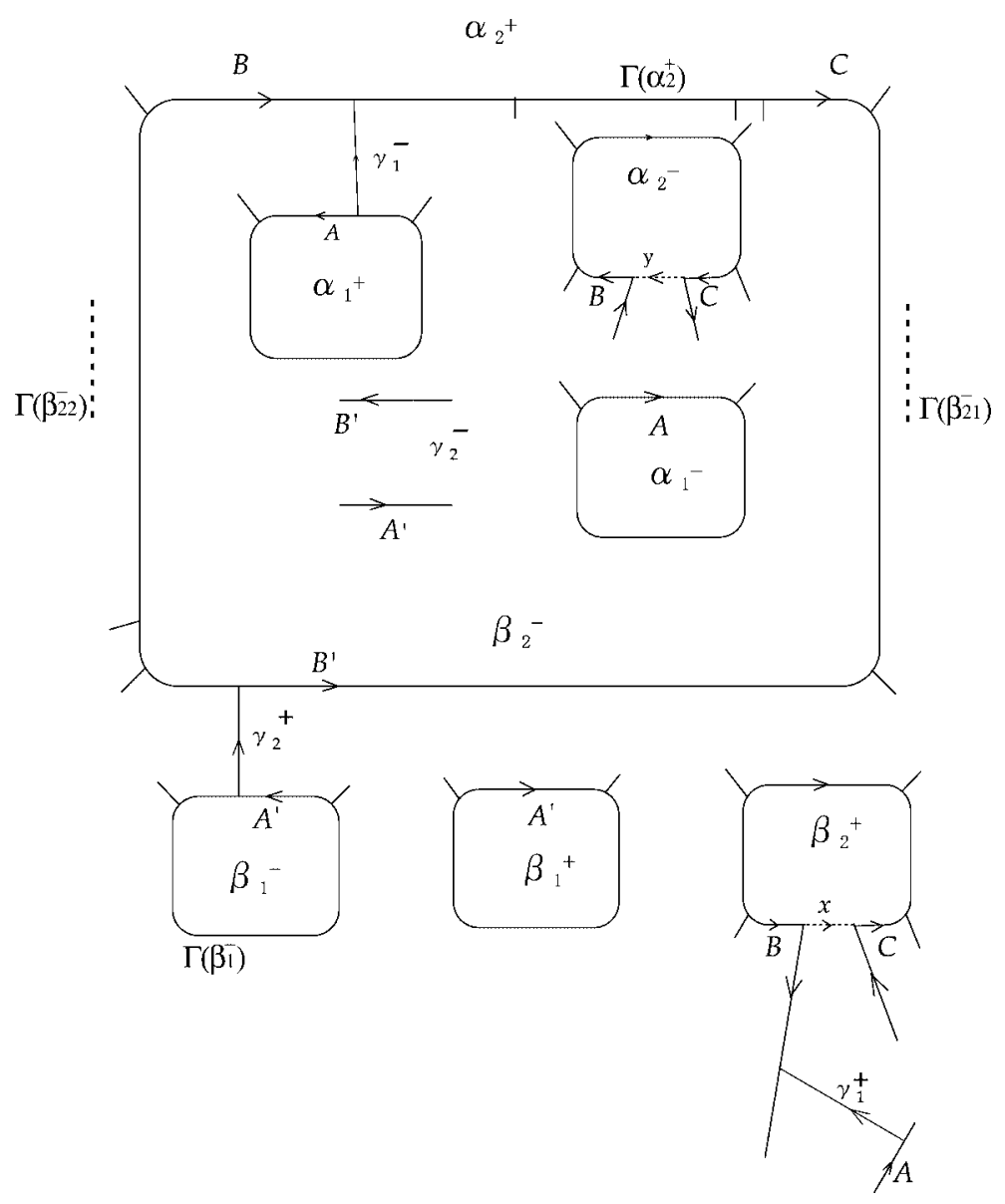

FIGURE 13 . The DS-diagram $\Delta_{0}$.

where

$$
\Gamma(B)=B_{1} V B_{*} Q_{1} S P_{2} B_{*} P_{1} T Q_{2} B_{*} U B_{2}
$$

and

$$
\begin{aligned}
\Gamma\left(B^{\prime}\right)= & \left(B_{2}^{\prime}\right)^{-1}\left(U^{\prime}\right)^{-1}\left(B_{*}^{\prime}\right)^{-1}\left(Q_{2}^{\prime}\right)^{-1}\left(T^{\prime}\right)^{-1}\left(P_{1}^{\prime}\right)^{-1}\left(B_{*}^{\prime}\right)^{-1}\left(P_{2}^{\prime}\right)^{-1} \\
& \left(S^{\prime}\right)^{-1}\left(Q_{1}^{\prime}\right)^{-1}\left(B_{*}^{\prime}\right)^{-1}\left(V^{\prime}\right)^{-1}\left(B_{1}^{\prime}\right)^{-1}
\end{aligned}
$$

Defining a mark-line $\gamma_{1}^{-}$from $A$ to $B_{*}$ (or $\gamma_{2}^{+}$from $A^{\prime}$ to $B_{*}^{\prime}$ ) in parallel with the pre markline $\gamma_{1}^{-}$(or respectively $\gamma_{2}^{+}$), we apply digging operations along mark-lines $\gamma_{1}^{-}$and $\gamma_{2}^{+}$. By Lemma 2.1 of [7], these digging operations connect the three cycles into only one cycle. Now 
we obtain a splittable DS-diagram $\Delta_{S}$ having a splitting cycle

$$
\Gamma_{1}=\left\{\Gamma_{1}(B, A) \Gamma\left(\alpha_{2}^{+}\right) C \Gamma\left(\beta_{21}^{-}\right) \Gamma_{1}\left(B^{\prime}, A^{\prime}\right) \Gamma\left(\beta_{22}^{-}\right)\right\},
$$

where

$$
\Gamma_{1}(B, A)=B_{1} V B_{b} E^{-1} B_{\sharp} Q_{1} S P_{2} B_{b} D_{\sharp}\left(A_{\sharp} \Gamma\left(\alpha_{1}^{+}\right) A_{b}\right) D_{b} B_{\sharp} P_{1} T Q_{2} B_{b} B B_{\sharp} U_{b} U U_{\sharp} B_{2}
$$

and

$$
\begin{aligned}
\Gamma_{1}\left(B^{\prime}, A^{\prime}\right)= & \left(B_{2}^{\prime}\right)^{-1}\left(U_{\sharp}^{\prime}\right)^{-1}\left(U^{\prime}\right)^{-1}\left(U_{b}^{\prime}\right)^{-1}\left(B_{\sharp}^{\prime}\right)^{-1}\left(B^{\prime}\right)^{-1}\left(B_{b}^{\prime}\right)^{-1}\left(Q_{2}^{\prime}\right)^{-1}\left(T^{\prime}\right)^{-1}\left(P_{1}^{\prime}\right)^{-1}\left(B_{\sharp}^{\prime}\right)^{-1} \\
& \left(D_{b}^{\prime}\right)^{-1}\left(\left(A_{b}^{\prime}\right)^{-1} \Gamma\left(\beta_{1}^{-}\right)\left(A_{\sharp}^{\prime}\right)^{-1}\right)\left(D_{\sharp}^{\prime}\right)^{-1} \\
& \left(B_{b}^{\prime}\right)^{-1}\left(P_{2}^{\prime}\right)^{-1}\left(S^{\prime}\right)^{-1}\left(Q_{1}^{\prime}\right)^{-1}\left(B_{\sharp}^{\prime}\right)^{-1} E^{\prime}\left(B_{b}^{\prime}\right)^{-1}\left(V^{\prime}\right)^{-1}\left(B_{1}^{\prime}\right)^{-1} .
\end{aligned}
$$

We apply Theorem 3.1 of [7] to the 1-labels $B_{\sharp}$ and $B_{\sharp}^{\prime}$.

$$
\Gamma_{2}=\left\{\Gamma_{2}(B, A) \Gamma\left(\alpha_{2}^{+}\right) C \Gamma\left(\beta_{21}^{-}\right) \Gamma_{2}\left(B^{\prime}, A^{\prime}\right) \Gamma\left(\beta_{22}^{-}\right)\right\},
$$

where

$$
\Gamma_{2}(B, A)=B_{1} V B_{b} E^{-1} L Q_{1} S P_{2} B_{b} D_{\sharp}\left(A_{\sharp} \Gamma\left(\alpha_{1}^{+}\right) A_{b}\right) D_{b} N P_{1} T Q_{2} B_{b} B M U_{b} U U_{\sharp} B_{2}
$$

and

$$
\begin{aligned}
\Gamma_{2}\left(B^{\prime}, A^{\prime}\right)= & \left(B_{2}^{\prime}\right)^{-1}\left(U_{\sharp}^{\prime}\right)^{-1}\left(U^{\prime}\right)^{-1}\left(U_{b}^{\prime}\right)^{-1}\left(M^{\prime}\right)^{-1}\left(B^{\prime}\right)^{-1}\left(B_{b}^{\prime}\right)^{-1}\left(Q_{2}^{\prime}\right)^{-1}\left(T^{\prime}\right)^{-1}\left(P_{1}^{\prime}\right)^{-1}\left(N^{\prime}\right)^{-1} \\
& \left(D_{b}^{\prime}\right)^{-1}\left(\left(A_{b}^{\prime}\right)^{-1} \Gamma\left(\beta_{1}^{-}\right)\left(A_{\sharp}^{\prime}\right)^{-1}\right)\left(D_{\sharp}^{\prime}\right)^{-1} \\
& \left(B_{b}^{\prime}\right)^{-1}\left(P_{2}^{\prime}\right)^{-1}\left(S^{\prime}\right)^{-1}\left(Q_{1}^{\prime}\right)^{-1}\left(L^{\prime}\right)^{-1} E^{\prime}\left(B_{b}^{\prime}\right)^{-1}\left(V^{\prime}\right)^{-1}\left(B_{1}^{\prime}\right)^{-1} .
\end{aligned}
$$

Remove two 2-gons with each of boundary circles $U_{b} D_{b}$ and $U_{b}^{\prime-1} D_{b}^{\prime-1}$ by applying elementary deformation $I^{-}$for each of 2-gons. The splitting cycle $\Gamma_{3}$ is

$$
\Gamma_{3}=\left\{\Gamma_{3}(B, A) \Gamma\left(\alpha_{2}^{+}\right) C \Gamma\left(\beta_{21}^{-}\right) \Gamma_{3}\left(B^{\prime}, A^{\prime}\right) \Gamma\left(\beta_{22}^{-}\right)\right\},
$$

where

$$
\Gamma_{3}(B, A)=B_{1} V B_{b} E^{-1} L Q_{1} S P_{2} B_{b} D_{\sharp}\left(A_{\sharp} \Gamma\left(\alpha_{1}^{+}\right) A_{b}\right) P_{1} T Q_{2} B_{b} B U U_{\sharp} B_{2}
$$

and

$$
\begin{aligned}
\Gamma_{3}\left(B^{\prime}, A^{\prime}\right)= & \left(B_{2}^{\prime}\right)^{-1}\left(U_{\sharp}^{\prime}\right)^{-1}\left(U^{\prime}\right)^{-1}\left(B^{\prime}\right)^{-1}\left(B_{b}^{\prime}\right)^{-1}\left(Q_{2}^{\prime}\right)^{-1}\left(T^{\prime}\right)^{-1}\left(P_{1}^{\prime}\right)^{-1} \\
& \left(\left(A_{b}^{\prime}\right)^{-1} \Gamma\left(\beta_{1}^{-}\right)\left(A_{\sharp}^{\prime}\right)^{-1}\right)\left(D_{\sharp}^{\prime}\right)^{-1} \\
& \left(B_{b}^{\prime}\right)^{-1}\left(P_{2}^{\prime}\right)^{-1}\left(S^{\prime}\right)^{-1}\left(Q_{1}^{\prime}\right)^{-1}\left(L^{\prime}\right)^{-1} E^{\prime}\left(B_{b}^{\prime}\right)^{-1}\left(V^{\prime}\right)^{-1}\left(B_{1}^{\prime}\right)^{-1} .
\end{aligned}
$$

For a pair of two 1-labels $B_{b}$ and $B_{b}{ }^{\prime}$, we apply DS-deformations according to Theorem 4.2 of [7]. Firstly, we apply an elementary deformation $I I^{+}$to $B_{b}{ }^{\prime}$. Then, $\mathcal{Z}^{+} \cap \mathcal{Z}^{-}$consists two cycles as

$$
\mathcal{Z}^{+} \cap \mathcal{Z}^{-}=\left\{\Gamma_{4}(B, A) \Gamma\left(\alpha_{2}^{+}\right) C \Gamma\left(\beta_{21}^{-}\right) \Gamma_{4}\left(B^{\prime}, A^{\prime}\right) \Gamma\left(\beta_{22}^{-}\right)\right\} \cup\{G H I\}
$$


where

$$
\Gamma_{4}(B, A)=B_{1} V B_{b} E^{-1} L Q_{1} S P_{2} B_{b} D_{\sharp}\left(A_{\sharp} \Gamma\left(\alpha_{1}^{+}\right) A_{b}\right) P_{1} T Q_{2} B_{b} B U U_{\sharp} B_{2}
$$

and

$$
\begin{aligned}
\Gamma_{4}\left(B^{\prime}, A^{\prime}\right)= & \left(B_{2}^{\prime}\right)^{-1}\left(U_{\sharp}^{\prime}\right)^{-1}\left(U^{\prime}\right)^{-1}\left(B^{\prime}\right)^{-1}\left(Q_{2}^{\prime}\right)^{-1}\left(T^{\prime}\right)^{-1}\left(P_{1}^{\prime}\right)^{-1}\left(A_{\mathrm{b}}^{\prime}\right)^{-1} \Gamma\left(\beta_{1}^{-}\right)\left(A_{\sharp}^{\prime}\right)^{-1} \\
& \left(D_{\sharp}^{\prime}\right)^{-1}\left(P_{2}^{\prime}\right)^{-1}\left(S^{\prime}\right)^{-1}\left(Q_{1}^{\prime}\right)^{-1}\left(L^{\prime}\right)^{-1} E^{\prime}\left(V^{\prime}\right)^{-1}\left(B_{1}^{\prime}\right)^{-1} .
\end{aligned}
$$

Secondly, we take a simple arc $\gamma^{+}$from $B_{b}$ to $H$ in $\mathcal{Z}^{+} \cdot \gamma^{+}$may intersects with $p$ 1-cells with 1-labels $W_{1}, W_{2}, \cdots W_{p-1}$ and $V$ where each $W_{i}$ is an edge inherited from the initial Heegaard diagram and $V$ is a new edge obtained by the deformations of remodeling, see Figure 14. The 1-label $W_{1}$ is an edge included in $\partial \beta_{1}$ and each $W_{i}(i=2,3 \cdots, p-1)$ is an edge included in $\partial \alpha_{1} \cup \partial \alpha_{2}$. Along $\gamma^{+}$as a mark-line, we apply a digging operation.

Lastly, applying an elementary deformation $I I^{+}$to $X_{1}$ and an elementary deformation $I^{+}$to $X_{2}$ (edges $X_{1}$ and $X_{2}$, see Figure 12-c in [7]), we obtain a DS-diagram $\Delta_{1}=(G, f, P ; e)$ with an E-cycle $e$. The splitting cycle $\Gamma_{e}$ which is the sequence of successive 1-labels on $e$ is

$$
\Gamma_{e}=\left\{\Gamma_{5}(B, A) \tilde{\Gamma}\left(\alpha_{2}^{+}\right) \Gamma(C) \Gamma\left(\beta_{21}^{-}\right) \Gamma_{5}\left(B^{\prime}, A^{\prime}\right) \Gamma\left(\beta_{22}^{-}\right)\right\},
$$

where

$$
\begin{aligned}
\Gamma_{5}(B, A)= & B_{1}\left(V_{b} V V_{\sharp} J_{1} X_{*} J_{2} E^{-1} L Q_{1} S P_{2} L_{1} Z L_{2} D_{\sharp}\right) A_{\sharp} \tilde{\Gamma}\left(\alpha_{1}^{+}\right) A_{b} \\
& \left(P_{1} T Q_{2} K_{1} T_{p} T_{p-1} \Gamma(T) T_{1} H_{1} I G H_{2} S_{1} \Gamma(S) S_{p-1} K_{2} B U U_{\sharp}\right) B_{2} \\
= & B_{1} \tilde{\Gamma}_{5}(B, A) B_{2}
\end{aligned}
$$

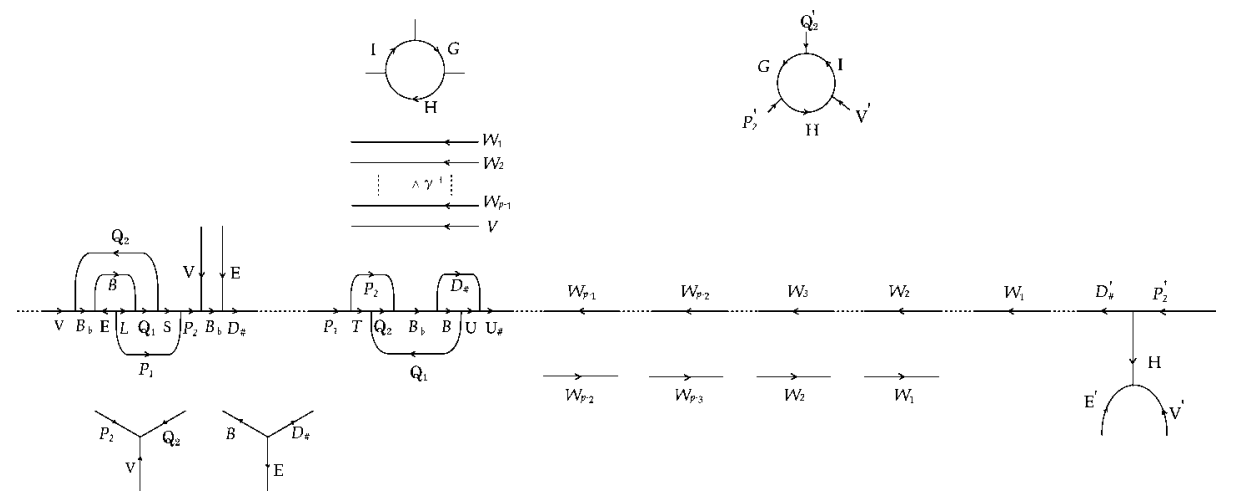

FIGURE 14. Mark line $\gamma^{+}$from $B_{b}$ to $H$. 
and

$$
\begin{aligned}
\Gamma_{5}\left(B^{\prime}, A^{\prime}\right)= & \left(B_{2}^{\prime}\right)^{-1}\left(U_{\sharp}^{\prime}\right)^{-1}\left(U^{\prime}\right)^{-1}\left(B^{\prime}\right)^{-1}\left(Q_{2}^{\prime}\right)^{-1}\left(T^{\prime}\right)^{-1}\left(P_{1}^{\prime}\right)^{-1}\left(A_{b}^{\prime}\right)^{-1} \tilde{\Gamma}\left(\beta_{1}^{-}\right)\left(A_{\sharp}^{\prime}\right)^{-1} \\
& \left(\left(D_{\sharp}^{\prime}\right)^{-1}\left(P_{2}^{\prime}\right)^{-1}\right)\left(S^{\prime}\right)^{-1}\left(Q_{1}^{\prime}\right)^{-1}\left(L^{\prime}\right)^{-1} E^{\prime}\left(V^{\prime}\right)^{-1}\left(B_{1}^{\prime}\right)^{-1} \\
= & \left(B_{2}^{\prime}\right)^{-1} \tilde{\Gamma}_{5}\left(B^{\prime}, A^{\prime}\right)\left(B_{1}^{\prime}\right)^{-1} .
\end{aligned}
$$

The consecutive sequence of 1-labels $\Gamma(T)=T_{p-2} \cdots T_{2}$ and $\Gamma(S)=S_{2} \cdots S_{p-2}$ included in $\Gamma_{5}(B, A)$, consist the new 1-labels which appear by the digging operation along $\gamma^{+}$, see Figure 15. If $\Gamma\left(\alpha_{i}^{+}\right)$contains the 1-label $W_{j}$ for some $j=2,3 \cdots p-1, \tilde{\Gamma}\left(\alpha_{i}^{+}\right)$includes the 1-labels $W_{j \sharp} W_{j} W_{j b}$. If $C=W_{k}$, for some $k=2,3 \cdots p-1$, then $\Gamma(C)=W_{k \sharp} W_{k} W_{k b}$. For $\Gamma\left(\beta_{1}^{-}\right)=\Gamma\left(\beta_{11}^{-}\right) W_{1} \Gamma\left(\beta_{12}^{-}\right), \tilde{\Gamma}\left(\beta_{1}^{-}\right)=\Gamma\left(\beta_{11}^{-}\right) W_{1 \sharp} W_{1} W_{1 b} \Gamma\left(\beta_{12}^{-}\right)$.

From now on, we will see the vertices on the E-cycle of the DS-diagram $\Delta_{1}$ in order to examine the E-data and to prove Lemma 4.2.1. The splitting cycle $\Gamma_{e}$ leads us to the E-data $\mathcal{E}\left(\Delta_{1}\right)$. We can write it as

$$
\mathcal{E}\left(\Delta_{1}\right)=\left\{\Psi_{B} \Lambda_{2}^{-} \Omega_{21}^{+} \Psi_{B^{\prime}} \Omega_{22}^{+}\right\}
$$

where

$$
\begin{aligned}
\Psi_{B}= & a_{1}^{-\ell} d_{p}^{-r} c_{p}^{-\ell} a_{2}^{-r} a_{3}^{+r} a_{4}^{+\ell} a_{5}^{-\ell} a_{6}^{-r} a_{7}^{+r} a_{8}^{+\ell} a_{9}^{-\ell} a_{10}^{-r} a_{2}^{+r} a_{5}^{+\ell} a_{11}^{-\ell} a_{12}^{-r} \Lambda_{1}^{-} \\
& a_{6}^{+r} a_{9}^{+\ell} a_{8}^{-\ell} a_{3}^{-r} a_{10}^{+r} c_{p}^{+\ell} \Upsilon_{1}^{+} c_{1}^{+r} b_{1}^{+r} b_{2}^{+r} b_{3}^{+r} d_{1}^{+\ell} \Upsilon_{2}^{+} d_{p}^{+r} a_{11}^{+\ell} a_{4}^{-\ell} a_{7}^{-r} a_{12}^{+r} a_{1}^{+\ell},
\end{aligned}
$$

and

$$
\Psi_{B^{\prime}}=b_{4}^{-\ell} b_{5}^{-r} b_{6}^{+r} b_{2}^{-r} b_{7}^{+\ell} b_{8}^{-\ell} b_{9}^{-r} \Omega_{11}^{+} c_{1}^{-r} d_{1}^{-\ell} \Omega_{12}^{+} b_{5}^{+r} b_{3}^{-r} b_{8}^{+\ell} b_{7}^{-\ell} b_{6}^{-r} b_{9}^{+r} b_{1}^{-r} b_{4}^{+\ell} .
$$

The subword $\Psi_{B}$ (or $\Psi_{B^{\prime}}$ ) consists of the vertices which are adjacent to the 1-labels of $\tilde{\Gamma}_{5}(B, A)$ (or $\tilde{\Gamma}_{5}\left(B^{\prime}, A^{\prime}\right)$, respectively). The subwords $\Lambda_{i}^{-}, \Omega_{i j}^{+}, \Upsilon_{1}^{+}$and $\Upsilon_{2}^{+}$consist of

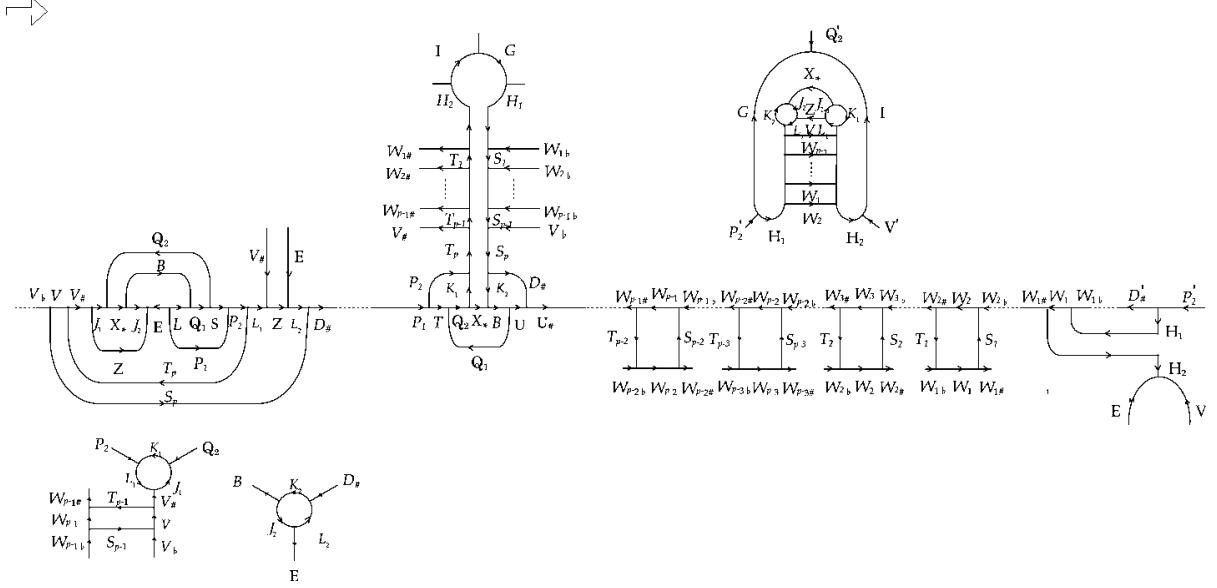

FIGURE 15. The DS-diagram $\Delta_{1}$ with E-cycle. 
the vertices which are adjacent to the 1-labels of $\tilde{\Gamma}\left(\alpha_{i}^{+}\right)\left(\right.$or $\tilde{\Gamma}\left(\alpha_{2}^{+}\right) W_{1 \sharp} W_{1}$, if $C=W_{k}$.), $\Gamma\left(\beta_{i j}^{-}\right), \Gamma(T)$ and $\Gamma(S)$, respectively. For the vertices included in $\Upsilon_{1}^{+}=c_{p-1}^{+} c_{p-2}^{+} \cdots c_{2}^{+}$ and $\Upsilon_{2}^{+}=d_{2}^{+} d_{3}^{+} \cdots d_{p-1}^{+}$, the vertices $c_{i}^{-}$and $d_{i}^{-}(i=2,3, \cdots p-1)$ are included in the sequence of 1-labels $W_{i \sharp} W_{i} W_{i b}$ on the E-cycle. This implies that $f\left(\Lambda_{1}^{-}\right) \cup f\left(\Lambda_{2}^{-}\right)=$ $f\left(\Omega_{11}^{+}\right) \cup f\left(\Omega_{12}^{+}\right) \cup f\left(\Omega_{21}^{+}\right) \cup f\left(\Omega_{22}^{+}\right) \cup f\left(\Upsilon_{1}^{+}\right) \cup f\left(\Upsilon_{2}^{+}\right)$. The vertices $a_{i}^{ \pm}(1 \leq i \leq 12)$, $b_{i}^{ \pm}(1 \leq i \leq 9), c_{i}^{ \pm}(1 \leq i \leq p)$ and $d_{i}^{ \pm}(1 \leq i \leq p)$ are new vertices which appear by deformations of remodeling. This shows that the E-data (15) is one required in Lemma 4.2.1

REMARK 1. By the moves from the coded sequence (15) to the coded sequence (14) in $\S 4.4$, each subword in (15) is inherited to the subwords in (14) as follows,

$$
\begin{aligned}
& \Psi_{B} \rightarrow W_{5,1}^{-} W_{5,2}^{+} W_{5,3}^{-} W_{5,4}^{+} W_{4,5}^{-} W_{4,7}^{-}, \\
& \Lambda_{2}^{-} \rightarrow \tilde{W}_{4,9}^{-}, \\
& \Omega_{21}^{+} \rightarrow \Omega_{21}^{+}=W_{5,10}^{+}, \\
& \Psi_{B^{\prime}} \rightarrow W_{5,11}^{-} W_{5,12}^{+} W_{5,13}^{-} W_{5,14}^{+} W_{5,15}^{-} W_{5,16}^{+} W_{5,17}^{-} W_{5,18}^{+} W_{5,19}^{-} b_{4}^{+\ell} \text { and } \\
& \Omega_{22}^{+} \rightarrow \Omega_{22}^{+} .
\end{aligned}
$$

\section{Difficulty for the case of $H G(M(\Delta))=3$}

In the main theorem, we have considered the case of $H G(M(\Delta))=2$. Here, we will mention briefly that the same method as in the case of $H G(M(\Delta))=2$ seems not to be applicable in the case of $H G(M(\Delta))=3$.

Let $\left(M_{1}, M_{2} ; \vec{D}_{1}, \vec{D}_{2}\right)$ be a Heegaard diagram with genus 3 , and let $\vec{D}_{1}=\left\{\alpha_{1}, \alpha_{2}, \alpha_{1}^{\prime}\right\}$ and $\vec{D}_{2}=\left\{\beta_{1}, \beta_{2}, \beta_{1}^{\prime}\right\}$ be complete meridian disk systems of $M_{1}$ and $M_{2}$ respectively. In order to apply the similar way of moves of the case $H G(M(\Delta))=2$, we have to prepare the following four pre mark-lines

$\gamma_{1}^{-}$with the initial point $p_{1} \in A_{1} \subset \partial \alpha_{1}{ }^{+}$and the terminal point $q_{1} \in B_{1} \subset \partial \alpha_{2}{ }^{+}$, $\gamma_{1}^{\prime-}$ with the initial point $p_{1}^{\prime} \in A_{1}^{\prime} \subset \partial{\alpha_{1}^{\prime}}^{+}$and the terminal point $q_{1}^{\prime} \in B_{1}^{\prime} \subset \partial \alpha_{2}{ }^{+}$, $\gamma_{2}^{+}$with the initial point $p_{2} \in A_{2} \subset \partial \beta_{1}^{-}$and the terminal point $q_{2} \in B_{2} \subset \partial \beta_{2}{ }^{-}$and $\gamma_{2}^{\prime+}$ with the initial point $p_{2}^{\prime} \in A_{2}^{\prime} \subset \partial \beta_{1}^{\prime-}$ and the terminal point $q_{2}^{\prime} \in B_{2}^{\prime} \subset \partial \beta_{2}^{-}$,

where

(1) these arcs do not intersect with each other,

(2) both of $\gamma_{2}^{+}$and $\gamma_{2}^{\prime+}$ intersect with neither edges $B_{1}$ nor $B_{1}^{\prime}$. 
We also obtain a DS-diagram $\Delta_{1}$ in a similar method to the case of genus 2 by making 2-gon near the mark-line $\gamma_{1}^{\prime-}$. The splitting cycle of $\Delta_{1}$ can be written as

$$
\begin{aligned}
\Gamma\left(\Delta_{1}\right)= & \Gamma_{5}\left(B_{1}, A_{1}\right) \tilde{\Gamma}\left(\alpha_{2}^{+}\right) \Gamma(C) \Gamma\left(\beta_{21}^{-}\right) \Gamma_{5}\left(B_{2}, A_{2}\right) \Gamma\left(\beta_{22}^{-}\right) \\
& \Gamma\left(\beta_{21}^{\prime}{ }^{-}\right) \Gamma_{5}\left(B_{2}^{\prime}, A_{2}^{\prime}\right) \Gamma\left(\beta_{22}^{\prime}{ }^{-}\right) \Gamma_{5}\left(B_{1}^{\prime}, A_{1}^{\prime}\right) \tilde{\Gamma}\left(\alpha_{2}^{\prime+}\right)
\end{aligned}
$$

and it leads us to the E-data

$$
\mathcal{E}\left(\Delta_{1}\right)=\Psi_{B_{1}} \Lambda_{2}^{-} \Omega_{21}^{+} \Psi_{B_{2}} \Omega_{22}^{+} \Omega_{21}^{\prime+} \Psi_{B_{2}^{\prime}} \Omega_{22}^{\prime+} \Psi_{B_{1}^{\prime}} \Lambda_{2}^{\prime-} \text {. }
$$

Applying the moves for reducing the block number to $\Delta_{1}$, we obtain a DS-diagram $\Delta^{\prime}$ with the coded sequence

$$
\begin{aligned}
\mathcal{E}\left(\Delta^{\prime}\right)= & \left(W_{5,1}^{-} W_{5,2}^{+} W_{5,3}^{-} W_{5,4}^{+} W_{4,5}^{-} W_{4,7}^{-}\right) \tilde{W}_{4,9}^{-} W_{5,10}^{+} \\
& \left(W_{5,11}^{-} W_{5,12}^{+} W_{5,13}^{-} W_{5,14}^{+} W_{5,15}^{-} W_{5,16}^{+} W_{5,17}^{-} W_{5,18}^{+} W_{5,19}^{-} b_{4}^{+\ell}\right) \Omega_{22}^{+} \\
& W_{5,10}^{\prime+}\left(W_{5,11}^{\prime-} W_{5,12}^{\prime+} W_{5,13}^{\prime-} W_{5,14}^{\prime+} W_{5,15}^{\prime-} W_{5,16}^{\prime+} W_{5,17}^{\prime-} W_{5,18}^{\prime+} W_{5,19}^{\prime-} b_{4}^{\prime+\ell}\right) \Omega_{22}^{\prime+} \\
& \left(W_{5,1}^{\prime-} W_{5,2}^{\prime+} W_{5,3}^{\prime-} W_{5,4}^{\prime+} W_{4,5}^{\prime-} W_{4,7}^{\prime-}\right) \tilde{W}_{4,9}^{\prime-} \\
= & \left(\prod_{k=1}^{2} W_{5,2 k-1}^{-} W_{5,2 k}^{+}\right)\left(\prod_{k=5}^{10} W_{5,2 k-1}^{-} W_{5,2 k}^{+}\right) \\
& W_{5,10}^{\prime+}\left(\prod_{k=6}^{10} W_{5,2 k-1}^{\prime-} W_{5,2 k}^{\prime+}\right)\left(\prod_{k=1}^{2} W_{5,2 k-1}^{\prime-} W_{5,2 k}^{++}\right) W_{5,9}^{\prime-}
\end{aligned}
$$

where $W^{\prime-}{ }_{5,9}=W_{4,5}^{\prime-} W_{4,7}^{\prime-} \tilde{W}_{4,9}^{\prime-}$ and $W_{5,20}^{\prime+}={b^{\prime}}_{4}^{+\ell} \Omega_{22}^{\prime}{ }^{+}$and each subwords of $\mathcal{E}\left(\Delta_{1}\right)$ are inherited to the subwords of $\mathcal{E}\left(\Delta^{\prime}\right)$ as follows,

$$
\begin{aligned}
& \Psi_{B_{1}} \rightarrow W_{5,1}^{-} W_{5,2}^{+} W_{5,3}^{-} W_{5,4}^{+} W_{4,5}^{-} W_{4,7}^{-}, \\
& \Lambda_{2}^{-} \rightarrow \tilde{W}_{4,9}^{-}, \\
& \Omega_{21}^{+} \rightarrow \Omega_{21}^{+}=W_{5,10}^{+}, \\
& \Psi_{B_{2}} \rightarrow W_{5,11}^{-} W_{5,12}^{+} W_{5,13}^{-} W_{5,14}^{+} W_{5,15}^{-} W_{5,16}^{+} W_{5,17}^{-} W_{5,18}^{+} W_{5,19}^{-} b_{4}^{+\ell}, \\
& \Omega_{22}^{+} \rightarrow \Omega_{22}^{+}, \\
& \Omega_{21}^{\prime} \rightarrow \Omega_{21}^{+}=W_{5,10}^{+}, \\
& \Psi_{B_{2}^{\prime}} \rightarrow W_{5,11}^{-} W_{5,12}^{+} W_{5,13}^{-} W_{5,14}^{\prime+} W_{5,15}^{\prime} W_{5,16}^{\prime} W_{5,17}^{\prime-} W_{5,18}^{\prime+} W_{5,19}^{\prime} b_{4}^{\prime}, \\
& \Omega_{22}^{\prime}+\Omega_{22}^{+}, \\
& \Psi_{B_{1}^{\prime}}^{+} \rightarrow W_{5,1}^{\prime} W_{5,2}^{\prime+} W_{5,3}^{\prime-} W_{5,4}^{+} W_{4,5}^{\prime-} W_{4,7}^{-}, \\
& \Lambda_{2}^{\prime} \rightarrow \tilde{W}_{4,9}^{\prime} .
\end{aligned}
$$

The subwords $W_{i, j}^{\varepsilon}$ and $W_{i, j}^{\prime \varepsilon}(\varepsilon=+$ or -$)$ consists of similar symbols and order to the subwords $W_{i, j}^{\varepsilon}$ of (14). 
The subword $\left(\prod_{k=5}^{10} W_{5,2 k-1}^{-} W_{5,2 k}^{+}\right) W_{5,10}^{\prime+}\left(\prod_{k=6}^{10} W_{5,2 k-1}^{\prime-} W_{5,2 k}^{\prime+}\right) W_{5,1}^{\prime-} W_{5,2}^{\prime+}$ can be deformed to the blocks $\Theta_{1}^{-}$and $\Theta_{2}^{+}$. But the other subword $W_{5,3}^{\prime-} W_{5,4}^{\prime+} W_{5,9}^{\prime-} W_{5,1}^{-} W_{5,2}^{+}$ $W_{5,3}^{-} W_{5,4}^{+}$can not be deformed to less blocks any more by the similar confirmation to the proof of Theorem 1. So we obtain a DS-diagram with block number 4. If we apply the different way of moves, we may obtain a DS-diagram with block number 3 . But it is difficult to find a method to do it now.

\section{References}

[1] Benedetti R. and Petronio C., Branched Standard Spines of 3-Manifolds, Springer LNM 1653 (1997).

[2] Endoh M. and Ishin I., A New Complexity for 3-Manifolds, to appear in Japanese J. Math.

[3] IKEDA H., Acyclic fake surfaces, Topology 10 (1971), 9-36.

[4] IKEDA H., Invitation to DS-diagrams, Kobe J. Math. 2 (1985), 169-186.

[ 5 ] IKedA H., DS-diagrams with E-cycle, Kobe J. Math. 3 (1986), 103-112.

[6] Ikeda H., Yamashita M. and Yokoyama K., Symbolic Description of Homeomorphisms on Closed 3manifolds, Kobe J. Math. 13 (1996), 69-115.

[ 7 ] Ikeda H., Yamashita M. and Yokoyama K., Remodeling a DS-diagram into one with E-cycle, Tokyo J. Math. 23 (2000), 113-135.

[ 8 ] IsHit I., Flows and spines, Tokyo J. Math. 9 (1986), 505-525.

[9] IsHII I., Moves for Flow-spines and Topological Invariants of 3-Manifolds, Tokyo J.Math. 15 (1992), 297312.

[10] IsHII I., Fake braid group and 3-manifolds, Geometry and Its Applications (edited by T. Nagano et al.), World Scientific (1993), 51-58.

[11] Ochiai M., Heegaard-Diagrams and Whitehead-Graphs, Math. Sem. Notes of Kobe Univ. 7 (1979), $573-$ 590 .

Present Address:

Department of Mathematics, Faculty of Science and Technology, Sophia University, KIOI-CHO, CHIYODA-KU, TOKYO 102-8554 JAPAN. 